\title{
B Cell-Intrinsic Role for IRF5 in TLR9/BCR-Induced Human B Cell Activation, Proliferation, and Plasmablast Differentiation
}

\author{
Saurav De ${ }^{1,2}$, Baohong Zhang ${ }^{3}$, Tiffany Shih ${ }^{2}$, Sukhwinder Singh ${ }^{4}$, Aaron Winkler ${ }^{5}$, \\ Robert Donnelly ${ }^{4}$ and Betsy J. Barnes ${ }^{2,6 *}$
}

${ }^{1}$ Rutgers Graduate School of Biomedical Sciences, Newark, NJ, United States, ${ }^{2}$ Center for Autoimmune Musculoskeletal and Hematopoietic Diseases, The Feinstein Institute for Medical Research, Manhasset, NY, United States, ${ }^{3}$ Clinical Genetics and Bioinformatics, Pfizer Inc., Cambridge, MA, United States, ${ }^{4}$ Department of Pathology and Laboratory Medicine, Rutgers Biomedical and Health Sciences, New Jersey Medical School, Newark, NJ, United States, ${ }^{5}$ Department of Inflammation and Immunology, Pfizer Inc., Cambridge, MA, United States, ${ }^{6}$ Rutgers Biomedical and Health Sciences, New Jersey Medical School-Cancer Center, Newark, NJ, United States

OPEN ACCESS

Edited by: Jen Anolik, University of Rochester, United States

Reviewed by:

Paolo Casali,

The University of Texas Health Science Center San Antonio, United States

Lee Ann Garrett-Sinha, University at Buffalo, United States

*Correspondence:

Betsy J. Barnes bbarnes1@northwell.edu

Specialty section: This article was submitted to B Cell Biology, a section of the journal Frontiers in Immunology

Received: 27 September 2017 Accepted: 15 December 2017 Published: 10 January 2018

Citation:

De S, Zhang B, Shin T, Singh S, Winkler A, Donnelly R and Barnes BJ (2018) B Cell-Intrinsic Role for IRF5 in

TLR9/BCR-Induced Human B Cell

Activation, Proliferation, and

Plasmablast Differentiation.

Front. Immunol. 8:1938. doi: 10.3389/fimmu.2017.01938
Upon recognition of antigen, $B$ cells undergo rapid proliferation followed by differentiation to specialized antibody secreting cells (ASCs). During this transition, B cells are reliant upon a multilayer transcription factor network to achieve a dramatic remodeling of the B cell transcriptional landscape. Increased levels of ASCs are often seen in autoimmune diseases and it is believed that altered expression of regulatory transcription factors play a role in this imbalance. The transcription factor interferon regulatory factor 5 (IRF5) is one such candidate as polymorphisms in IRF5 associate with risk of numerous autoimmune diseases and correlate with elevated IRF5 expression. IRF5 genetic risk has been widely replicated in systemic lupus erythematosus (SLE), and loss of Irf5 ameliorates disease in murine lupus models, in part, through the lack of pathogenic autoantibody secretion. It remains unclear, however, whether IRF5 is contributing to autoantibody production through a B cell-intrinsic function. To date, IRF5 function in healthy human B cells has not been characterized. Using human primary naive B cells, we define a critical intrinsic role for IRF5 in B cell activation, proliferation, and plasmablast differentiation. Targeted IRF5 knockdown resulted in significant immunoglobulin (lg) D retention, reduced proliferation, plasmablast differentiation, and IgG secretion. The observed decreases were due to impaired B cell activation and clonal expansion. Distinct from murine studies, we identify and confirm new IRF5 target genes, IRF4, ERK1, and MYC, and pathways that mediate IRF5 B cell-intrinsic function. Together, these results identify IRF5 as an early regulator of human B cell activation and provide the first dataset in human primary B cells to map IRF5 dysfunction in SLE.

Keywords: interferon regulatory factor 5 , human primary B cells, plasmablasts, autoantibodies, immunoglobulin G, differentiation, toll-like receptor

\section{INTRODUCTION}

Antibody secreting cells (ASCs), referred to as plasmablasts and plasma cells, are critical mediators of adaptive immunity and are often found elevated in the circulation of patients with systemic lupus erythematosus (SLE) (1-4). ASCs release high titers of antibody capable of neutralizing invading antigen and stem from naive and memory $\mathrm{B}$ cells that have been activated through antigen recognition. Differentiation of naive B cells to ASCs requires multiple B cell activation pathways, 
including B cell receptor (BCR), T cell-mediated cytokine signaling, and toll-like receptors (TLRs) $(5,6)$. BCR activation occurs through binding of cognate antigen, leading to activation of distal signaling cascades, including the Ras-Raf-MEK-ERK1/2 kinase cascade and the Btk-PI3K-PLC-NFkB cascade, ultimately culminating in transcriptional activation of proliferation and prosurvival genes $(7,8)$. Complimentary $\mathrm{T}$ cell activation allows for interaction between $\mathrm{B}$ and $\mathrm{T}$ cells. Engagement of the respective $\mathrm{B}$ and $\mathrm{T}$ cell surface proteins, CD40 and CD40 ligand (CD40L), further enhances transcription of B cell survival and proliferation genes. T cell-mediated cytokine signaling acts to drive ASC differentiation, as well as to specify the antibody subclass secreted. TLR signaling can act synergistically with both $\mathrm{T}$ cell-dependent and -independent $\mathrm{B}$ cell activation pathways and represents a unique bridge between innate and adaptive immune responses (9).

These various B cell activation pathways utilize a network of transcription factors to drive proliferation, survival and differentiation (3). Immediately following activation, B cells rapidly proliferate to ensure sufficient numbers of antigen-specific B cells to undergo ASC differentiation. This initial burst of proliferation is dependent on the transcription factor MYC $(10,11)$. Following several rounds of proliferation, B cells upregulate interferon regulatory factor 4 (IRF4), BLIMP1, and XBP1, which control ASC differentiation and prepare the cell for antibody secretion (3, 12-14). In response to IRF4 upregulation, B cells undergo terminal chromosomal rearrangement of the immunoglobulin (Ig) locus known as class switch recombination (CSR) (12). CSR results in the switching of antibody subtype from IgM to IgG, $\operatorname{IgA}$, or IgE, and is dependent on the enzyme activation-induced deaminase (AID). The majority of work on ASC differentiation has occurred in mice as human primary B cells are notoriously difficult to manipulate (by knockdown or overexpression) for functional analyses.

Genetic variants in or near the transcription factor interferon regulatory factor 5 (IRF5) have been robustly associated with SLE risk and elevated IRF5 expression and activation have been reported in SLE immune cells (15-19). Mice lacking Irf5 are protected from murine lupus disease onset and severity (20-23). A common finding between the different models of murine lupus that lack Irf5 is the significant decrease in pathogenic autoantibody secretion suggesting a role for IRF5 in B cells. In mice, Irf5 was found to regulate IL6, PRDM1, and IgG2a expression $(22,24-26)$. SLE pathogenesis is associated with polyclonal B cell hyperreactivity resulting in an autoreactive B cell repertoire, elevated circulating ASCs and autoantibodies $(2,27)$. Whether IRF5 contributes to ASC differentiation or antibody production in human primary B cells is not known.

Here, we developed a method of targeted gene knockdown in human primary naive B cells. While IRF5 expression and activity have been well-characterized in human monocytes and dendritic cells, its role in B cells remains to be defined $(19,28-35)$. We show that IRF5 is required in the early stages of B cell activation and proliferation in response to TLR9/BCR-induced ASC differentiation. IRF5 knockdown resulted in a significant increase in the number of $\mathrm{IgD}^{+} \mathrm{B}$ cells, reduced activation, clonal expansion, plasmablast differentiation, and IgG1/3 secretion. Distinct from murine studies, we identify and confirm new IRF5 target genes, IRF4, ERK1, and MYC, that mediate IRF5 B cell-intrinsic function.

\section{MATERIALS AND METHODS}

\section{Naive B Cell Isolation}

Blood was drawn via peripheral phlebotomy and PBMC isolated by Ficoll centrifugation (18). PBMC were diluted to a concentration of $5 \times 10^{7}$ cells $/ \mathrm{mL}$ and naive $\mathrm{B}$ cells isolated using Stem Cell Technologies Kit (Cat\#: 19254). Magnetic separation was performed to achieve a $>95 \%$ enriched population of naive $\mathrm{B}$ cells $\left(\mathrm{CD} 19^{+} \mathrm{CD} 20^{+} \mathrm{IgD}^{+} \mathrm{CD} 27^{-}\right)$, as determined by flow cytometry (Figure S1A in Supplementary Material). This study was carried out in accordance with the recommendations of the Rutgers Biomedical and Health Sciences IRB and the Feinstein Institute for Medical Research IRB with written informed consent from all subjects. All subjects gave written informed consent in accordance with the Declaration of Helsinki. The protocol was approved by the Rutgers Biomedical and Health Sciences IRB and the Feinstein Institute for Medical Research IRB. The Ramos lymphoblastic B cell line was purchased from ATCC $^{\circledR}$ and cultured in RPMI-1640 with $10 \%$ fetal bovine serum.

\section{Imaging Flow Cytometry Analysis of IRF5 Activation}

Isolated PBMC were stained for CD19 (BD Biosciences \#562847) and fixed overnight in $1 \%$ paraformaldehyde. Cells were permeabilized the following day in $0.01 \%$ Triton-X-100 and stained for intracellular IRF5 (Abcam \#ab193245) (19). Images were acquired on the Amnis ImageStream X Mark II imaging flow cytometer using the $40 \times$ objective. Nuclear translocation was quantified in the Amnis IDEAS software suite using the similarity score feature (Figure S1B in Supplementary Material).

\section{IRF5 siRNA Nucleofection}

Isolated naive $\mathrm{B}$ cells $\left(3 \times 10^{6}\right)$ were resuspended in Amaxa buffer P3 (Lonza: \#V4XP-3032) and distributed to Amaxa $100 \mu \mathrm{L}$ cuvettes. B cells were nucleofected with $500 \mathrm{nM}$ of mock, ON-TARGETplus non-targeting control pool (GE Dharmacon: \#D-001810-10-05), or SMARTpool ON-TARGETplus human IRF5 siRNA (GE Dharmacon: \#L-011706-00-0010). Cells were nucleofected on the Amaxa 4D Nucleofector using program EO-117 and then immediately added to $1 \mathrm{~mL}$ of RPMI 1640 ( $+10 \%$ FBS, $1 \times$ glutamine, $1 \times$ non-essential amino acids $)$ and cultured for $24 \mathrm{~h}$, pelleted and re-nucleofected with siRNA. For GFP co-nucleofection, pmaxGFPTM Vector (Lonza) or GFP mRNA (Trinity Biotech: \#L6101) was titrated over a concentration range with $500 \mathrm{nM} I R F 5$ siRNA; $15 \mu \mathrm{g}$ GFP mRNA gave the best results.

\section{qRT-PCR and Western Blotting}

RNA was isolated with Trizol $^{\circledR}$ and qRT-PCR performed as described (18) with primer sets: MYC 5'-CCTGGTGCTCCATG AGGAGAC, 3'-CAGACTCTGACCTTTTGCCAGG; IRF4 5'-G 
AACGAGGAGAAGAGCATCTTCC, $3^{\prime}$-CGATGCCTTCTCGG AACTTTCC; IL65' -AGACAGCCACTCACCTCTTCAG, 3' -TT CTGCCAGTGCCTCTTTGCTG; PRDM1 5'-AGAAGGCTC CAGCCATCTCTGT, 3 '-TGCTGGTAGAGTTCGGTGCAGA. Threshold values $\left(C_{\mathrm{T}}\right)$ were averaged over each sample replicate, followed by normalization via the $\Delta \Delta C_{\mathrm{T}}$ method to $\beta$-actin. For Western blot analysis, 2 days postnucleofection, naive $\mathrm{B}$ cells were stimulated with mock or anti-IgM ${ }^{+} \mathrm{CpG}-\mathrm{B}$ for $24 \mathrm{~h}$ and then harvested for lysate preparation in RIPA buffer $(10 \mathrm{nM}$ Tris-HCl pH 8.0, 1 mM EDTA, 1\% Triton X-100, 0.1\% Sodium Deoxycholate, $0.1 \%$ SDS, and $140 \mathrm{mM} \mathrm{NaCl}$ ) (36).

\section{In Vitro B Cell Activation and Plasmablast Differentiation}

Isolated naive $B$ cells were cultured in 96-well U-bottom plates at a minimal density of $1 \times 10^{6}$ with either $150 \mathrm{ng} / \mathrm{mL}$ CD $40 \mathrm{~L}$ (Peprotech \#310-02) alone or with $100 \mathrm{ng} / \mathrm{mL}$ IL21 (Peprotech \#200-21), $10 \mu \mathrm{g} / \mathrm{mL}$ anti-IgM antibody (Southern Biotech \#2020-01), and $2.5 \mu \mathrm{g} / \mathrm{mL}$ CpG-B (Hycult Tech \#HC4039). For plasmablast differentiation, isolated naive B cells were cultured for 7 days in the presence of stimulating cocktail.

\section{Flow Cytometry Analysis}

Isolated B cells were washed and stained with Live/Dead viability discrimination dye (Life Tech \#L34968). Cells were subsequently blocked in 2\% BSA supplemented with Fc Blocker (BioLegend) for $15 \mathrm{~min}$ and then stained with antibodies against B cell surface makers for $1 \mathrm{~h}$ [all antibodies were from BD Biosciences except CD38-PE/Texas Red (Life Technologies \#MHCD3817); CD19-BV510, \#562847; CD20-BUV396, \#563782; CD27-BV421, \#560448; IgD-APC, \#348222; IgM-PerCP/Cy5.5, \#314512; CD138-PE, \#552026; IgG-PE/Cy7, \#409316; CD45-APC/Cy7, \#368516]. After staining, cells were washed two times in PBS without $\mathrm{Mg}^{++}$or $\mathrm{Ca}^{2+}$ and then fixed in 2\% PFA before analysis on a BD Fortessa or BD LSR flow cytometer. Plasmablasts were defined as $\mathrm{CD} 19^{+} \mathrm{CD} 20^{+} \mathrm{IgD}^{-} \mathrm{CD} 27^{+} \mathrm{CD} 38^{+} \mathrm{B}$ cells. B cell activation was determined with CD86 surface staining (\#562432). Ig production was determined by intracellular staining with anti-IgA (Life Tech \#Z25002), anti-IgE (Biolegend \#325510), and anti-IgG antibodies in plasmablasts; AID was detected with antibody \#Z25302 (Life Tech). For intracellular IRF5 staining, after overnight fixation, cells were permeabilized the following day in $0.1 \%$ Triton X-100 and rinsed in PBS $2 \times$ before blocking in $2 \%$ BSA solution. IRF5 staining was performed using antiIRF5 antibody conjugated to Alexa Fluor 488 (Abcam Catalog\#: AB193245).

\section{IgG Isotype ELISA}

Secretion of IgG isotypes was determined in media from 7-day cultures by ELISA (ThermoFisher \#991000), as per manufacturer instructions.

\section{Proliferation Assay}

Nucleofected cells were stained with $2.5 \mu \mathrm{M}$ CFSE proliferation dye (Life Tech \#C34554) or Cell Trace Violet dye (Life Tech \#C34557) for $20 \mathrm{~min}$. Cells were then washed, plated, and stimulated for 5 days. Proliferation was analyzed in Live/ Dead $^{-} \mathrm{CD} 19^{+} \mathrm{CD} 20^{+} \mathrm{CD} 38^{\text {lo }} \mathrm{B}$ cells.

\section{IRF5 ChIP-Seq}

Primary naive $B$ cells were isolated as previously outlined and plated at a density of $1 \times 10^{6} / \mathrm{mL}$. B cells were either mock or anti-IgM+ $\mathrm{CpG}-\mathrm{B}$ stimulated for $4 \mathrm{~h}$ at $37^{\circ} \mathrm{C}$ in Iscove's Modified Dulbecco's Media (Thermo Fisher) supplemented with 10\% FBS. Cells were subsequently washed $2 \times$ in PBS- $\mathrm{Ca}^{2+}-\mathrm{Mg}^{2+}$ and fixed in $1 \%$ methanol-free paraformaldehyde for $10 \mathrm{~min}$ at $37^{\circ} \mathrm{C}$. The cross-linking reaction was quenched by the addition of glycine to a final concentration of $0.125 \mathrm{M}$. Cells were then washed $2 \times$ in PBS- $\mathrm{Ca}^{2+}-\mathrm{Mg}^{2+}$ and the final cell pellet lysed in $300 \mu \mathrm{L}$ of RIPA lysis buffer. Lysates were loaded into a Covaris sonication microtube and sonicated in a Covaris S2 immersion sonicator with a duty cycle of $5 \%$, intensity 2 , at 200 burst for 15 cycles at $1 \mathrm{~min}$ each. Lysates were then precleared with $75 \mu \mathrm{L}$ of a $50 \%$ agarose protein a/g bead slurry for $1 \mathrm{~h}$. IRF5 immunoprecipitation (IP) was performed overnight at $4^{\circ} \mathrm{C}$ on precleared lyates using $4 \mu \mathrm{g}$ of validated IRF5 antibody (Abcam\#: ab124792) (34). A 40\% protein a/g bead slurry was added to each IP for $3 \mathrm{~h}$ to allow conjugation of IRF5 antibody to beads. IP bead samples were then washed $3 \times$ in each of the following buffers in order-RIPA lysis buffer, $\mathrm{LiCl}$ wash buffer $(100 \mathrm{mM}$ Tris pH 7.5, $500 \mathrm{mM} \mathrm{LiCl}$, $1 \%$ NP-40, 1\% sodium deoxycholate), and TE buffer (10 mM Tris-HCl pH 7.5, 10 mM Na 2 EDTA). IP Beads were incubated overnight in elution buffer $\left(1 \% \mathrm{SDS} / 0.1 \mathrm{M} \mathrm{NaHCO}_{3}\right)$ at $65^{\circ} \mathrm{C}$ to reverse cross-links. Eluted DNA was then treated with RNAse $(0.5 \mathrm{mg} / \mathrm{mL})$ and Proteinase $\mathrm{K}(10 \mathrm{mg} / \mathrm{mL})$ for $2 \mathrm{~h}$ each before isolation using phenol chloroform extraction. Briefly, an equal volume of phenol chloroform was added to each sample, followed by vigorous vortexing. The mixture was then transferred to a phase lock tube, spun for $5 \mathrm{~min}$ at max speed, and the aqueous phase removed. Repeated ethanol precipitation was used to isolate final DNA. Resulting ChIP DNA was quantified by bioanalyzer to ensure sufficient yield and proper fragmentation. Samples were submitted to the New York University (NYU) Genome Center for library preparation and single-end sequencing on Illumina HiSeq to a read depth of 50 million reads. Ramos B cell samples were submitted to the Rutgers NJMS Genomic Sequencing Core for ChIP-Seq.

\section{RNA-Seq of Primary Human B Cells}

Isolated primary B cells nucleofected with mock, scrambled, or IRF5 siRNA were mock or anti-IgM ${ }^{+} \mathrm{CpG}$ stimulated for $6 \mathrm{~h}$ at $37^{\circ} \mathrm{C}$. RNA was purified using the Qiagen RNeasy isolation kit and on-column DNA digestion performed to remove genomic DNA. Final RNA was eluted in $30 \mu \mathrm{L}$ RNAse-free water and submitted to the NYU Genome Center for single end sequencing on Illumina HiSeq to a depth of 20 million reads in the case of primary B cells and 40 million reads for Ramos B cells.

\section{Bioinformatics Analysis of ChIP-Seq and RNA-Seq Data}

ChIP-Seq data was processed by HiChIP (37). Reads were mapped to the Hg19 human reference sequences using BWA 
at default parameters (38). Peaks were called using the MACS2 algorithm at the following parameters $(p=0.0001, m=10.30)$ and using IgG isotype controls as input against IRF5 ChIP treated samples. Motif enrichment analysis was performed using the TomTom Motif Comparison suite (MEME Suite 4.8) (39). Peaks were visualized using IGV genome browser (40). RNA-Seq data QC, read mapping by STAR (41), read counting by featureCounts (42) were handled through the QuickRNASeq pipeline developed at Pfizer (43). Subsequently, EdgeR was used to determine differentially expressed genes; a $p$-value $\leq 0.05$ and a false discovery rate (FDR) $<0.05$ after Benjamin-Hochberg correction was used for determining significant differential gene expression (44). Normalized RNA-Seq data are presented as reads per kilobase of transcript per million reads mapped (RPKM) (45). The QC report, processed read count table, RPKM table, and interactive data exploring tool generated by QuickRNAseq is available at https://baohongz.github.io/IRF5_knockdown. We used HOMER (46) for pathway analysis as it contains a program for performing functional enrichment analysis from a list of genes (http:// homer.ucsd.edu/homer/microarray/go.html) HOMER uses a one-sided Fisher's exact test to determine the significance of over-representation of a gene set in the input list. We focused our analysis on enriched pathway gene sets from WikiPathways (47) as it is the most comprehensive open source pathway collection. A more stringent cutoff of log2 fold-change $\geq 1$ and FDR $\leq 0.001$ was applied to select differentially expressed genes before performing the enrichment analysis to minimize the impact of false positives due to the small sample size.

\section{Statistical Analyses}

Unless otherwise stated, one-way ANOVA or two-way ANOVA were used to compare means among three or more independent groups. Bonferroni posttest to compare all pairs of data sets was determined when overall $p$-value was $<0.05$. All statistical analyses were performed using GraphPad Prism (version 7.0). Data are reported as mean $\pm \mathrm{SD}$. In each figure legend, the number (n) of biological repeats included in the final statistical analysis is indicated. $p$-value $<0.05$ was considered significant.

\section{RESULTS}

\section{TLR9/BCR Stimulation Induces IRF5 Nuclear Translocation}

Among the pathways known to play a role in ASC differentiation is TLR signaling (48). IRF5 acts downstream of TLRs in monocytes and dendritic cells, but characterization of IRF5 activation in human B cells has not been shown. IRF5 resides in the cytoplasm of unstimulated cells and upon activation translocates to the nucleus (49-51). We examined IRF5 activation following treatment of healthy $\mathrm{CD} 19^{+} \mathrm{B}$ cells with activating stimuli using imaging flow cytometry (gating in Figure S1B in Supplementary Material). We failed to detect significant IRF5 nuclear translocation with BCR activating anti-IgM antibody or T cell-dependent CD40L but detected significant activation with the TLR9 agonist CpG-B (Figures 1A,B). As the majority of peripheral B cells are antigen naive and express low levels of TLR9, we combined
BCR stimulation, known to upregulate TLR9, with CpG-B and examined IRF5 activation (52). Anti-IgM plus CpG-B also provided a significant increase in IRF5 nuclear translocation over mock (Figures 1A,B). Since ASC differentiation can utilize T cell-mediated signals, we examined IRF5 activation following stimulation with CD40L and IL21. Although we did not detect an increase in IRF5 activation with CD40L and IL21, the combination of CD40L, anti-IgM, IL21, and CpG-B significantly increased IRF5 nuclear translocation to levels seen with anti-IgM and CpG-B (Figures 1A,B).

In murine monocytes, IRF5 nuclear translocation was shown to increase linearly over time following stimulation (24). We measured IRF5 activation kinetics in human B cells following anti-IgM ${ }^{+}$CpG-B stimulation. Significant IRF5 nuclear translocation was first observed at $1 \mathrm{~h}$, with peak translocation occurring at $4 \mathrm{~h}$ (Figure 1C). To determine if activation is a byproduct of increased expression, we measured IRF5 protein levels in total $\mathrm{B}$ cells following $2 \mathrm{~h}$ stimulation; no significant difference in IRF5 levels was detected (Figures S1C,D in Supplementary Material). Although IRF5 expression was unchanged at this early time point of activation, IRF5 expression across B cell subsets may differ and has not previously been measured. In monocytes, increased IRF5 expression is known to be deterministic of subset fate, with higher IRF5 levels seen in inflammatory M1 macrophages (33). To determine if IRF5 expression has similar traits in B cells, we quantified expression in B cell subsets from healthy donors. Routine vaccination is known to increase the occurrence of plasmablasts and plasma cells in the periphery 7 days post-immunization (53). We therefore utilized peripheral blood from individuals immunized with the flu vaccine to define IRF5 protein levels in $\mathrm{CD}_{19}{ }^{+} \mathrm{CD} 20^{+}$naive $\left(\mathrm{IgD}^{+} \mathrm{CD} 38^{-} \mathrm{CD} 27^{-} \mathrm{CD} 24^{-}\right)$, transitional $\left(\mathrm{IgD}^{+} \mathrm{CD} 38^{+} \mathrm{CD} 27^{-} \mathrm{CD} 24^{+}\right)$, non-switched memory $\left(\mathrm{IgD}^{+} \mathrm{CD} 3\right.$ $\left.8^{-} \mathrm{CD} 27^{+} \mathrm{CD} 24^{-}\right)$, switched memory $\left(\mathrm{IgD}^{-} \mathrm{CD} 27^{+} \mathrm{CD} 24^{-}\right)$, plasmablasts $\left(\mathrm{IgD}^{-} \mathrm{CD} 38^{\mathrm{hi}} \mathrm{CD} 27^{+} \mathrm{CD} 24^{-} \mathrm{CD} 138^{-}\right)$, and plasma cells $\left(\mathrm{IgD}^{-} \mathrm{CD} 38^{\text {hi }} \mathrm{CD} 27^{+} \mathrm{CD} 24^{-} \mathrm{CD} 138^{+}\right)$(Figure 1D). Naive and transitional B cells expressed lower levels of IRF5 protein, whereas both non-switched and switched memory had increased expression. Plasmablasts and plasma cells had the highest IRF5 expression (Figures 1E,F). Similar findings were made in blood from healthy, non-vaccinated individuals (Figures S1E,F in Supplementary Material). These data suggest that IRF5 may regulate both B cell subset fate and effector function in mature B cell populations.

\section{IRF5 Knockdown Reduces ASC Differentiation}

To investigate the role of IRF5 in human ASC differentiation, we validated and optimized targeted IRF5 knockdown in human primary naive B cells using nucleofection $(36,54,55)$. Isolated naive $\mathrm{B}$ cells were nucleofected with mock, ON-TARGETplus non-targeting control pool (scrambled), or SMARTpool ON-TARGETplus human IRF5 siRNA. IRF5 knockdown efficiency was quantified by measuring IRF5 transcript and protein levels. A 50\% reduction in IRF5 transcript levels was detected $24 \mathrm{~h}$ postnucleofection in comparison to mock and scrambled (Figure 2A). To ensure that a reduction in transcripts correlated with a reduction in IRF5 protein levels, protein lysates were 


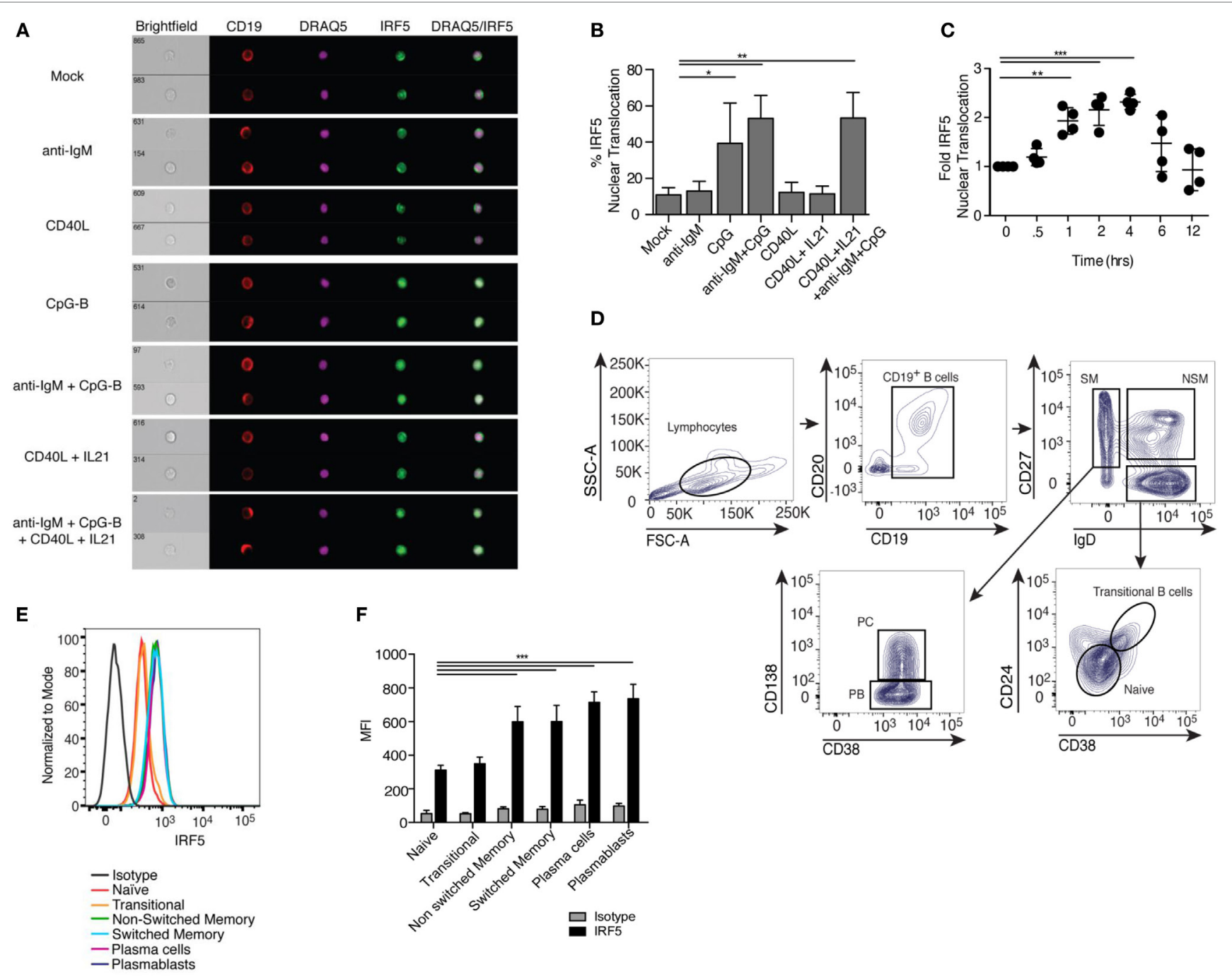

FIGURE 1 | Toll-like receptor 9/B cell receptor stimulation induces Interferon regulatory factor 5 (IRF5) nuclear translocation. (A) Representative images of IRF5 cellular localization in human CD19+ B cells from a single healthy donor that were stimulated with mock, anti-immunoglobulin (Ig) M antibody, CD40 ligand (CD4OL), CpG-B, or CD4OL and IL21, and the combination of anti-IgM antibody, CD4OL, IL21, and CpG-B for $2 \mathrm{~h}$. PBMC were surface-stained with anti-CD19 antibodies, fixed and permeabilized, then stained for intracellular IRF5 and nuclear DRAQ5. Samples were then subjected to imaging flow cytometry followed by analysis in the IDEAS software suite. (B) Frequency of cells in (A) with IRF5 nuclear translocation, which was defined by an IRF5 and DRAQ5 similarity score $\geq 2$ (one-way ANOVA with Tukey's post hoc test; $n=4$ independent donors). (C) IRF5 nuclear translocation was quantified over $12 \mathrm{~h}$ in isolated B cells following stimulation with anti-lgM+ CpG-B. Data was normalized to mock to minimize donor variability (one-way ANOVA with Tukey's post hoc test; $n=4$ independent donors). (D) Representative gating strategy for human $\mathrm{B}$ cells subsets in peripheral blood of healthy donors who received the influenza vaccine 7 days prior to phlebotomy. B cell populations were defined as $\mathrm{CD} 19^{+} \mathrm{CD} 20^{+}$naive $\left(\operatorname{lgD}{ }^{+} \mathrm{CD} 38^{-} \mathrm{CD} 27^{-} \mathrm{CD} 24^{-}\right)$, transitional $\left(\mathrm{lgD}^{+} \mathrm{CD} 27^{-} \mathrm{CD} 38^{+} \mathrm{CD} 24^{+}\right)$, non-switched memory $\left(\mathrm{NSM}^{-} \operatorname{lgD}^{+} \mathrm{CD} 27^{+} \mathrm{CD} 38^{-} \mathrm{CD} 24^{-}\right)$,

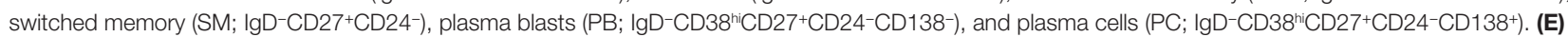
Representative histograms of IRF5 protein expression in B cell subsets gated in (D). (F) Average MFI of IRF5 expression in gated B cell subsets (two-way ANOVA with Tukey's multiple comparison post hoc test; $n=5$ independent donors). Error bars represent SD. ${ }^{*} p \leq 0.05 ;{ }^{* \star} p \leq 0.01 ;{ }^{* \star} p \leq 0.001$.

prepared at $72 \mathrm{~h}$ postnucleofection (with $24 \mathrm{~h}$ anti-IgM plus CpG-B stimulation) and analyzed by Western blot and flow cytometry (Figures 2B-D). Costimulation provided an increase in IRF5 expression levels over mock unstimulated, and these levels were significantly reduced following nucleofection with IRF5 siRNA, while IRF5 levels remained steady in mock and scrambled controls supporting the specificity of knockdown. Notably, analysis by flow cytometry showed reduced IRF5 expression amongst the total population of viable B cells in comparison to scrambled control. Stimulation with anti-IgM and CpG-B did not impact IRF5 knockdown efficacy (Figures 2A,B).

Interferon regulatory factor 5 is known to regulate the transcription of pro-apoptotic genes following DNA damage or death receptor signaling $(56,57)$. To quantify B cell viability following IRF5 knockdown, nucleofected naive B cells were stained with tryphan blue $24 \mathrm{~h}$ after mock or anti-IgM and CpG-B stimulation. Although nucleofection itself results in $\sim 15 \%$ reduction in cell viability, no further decrease in viability was found after IRF5 

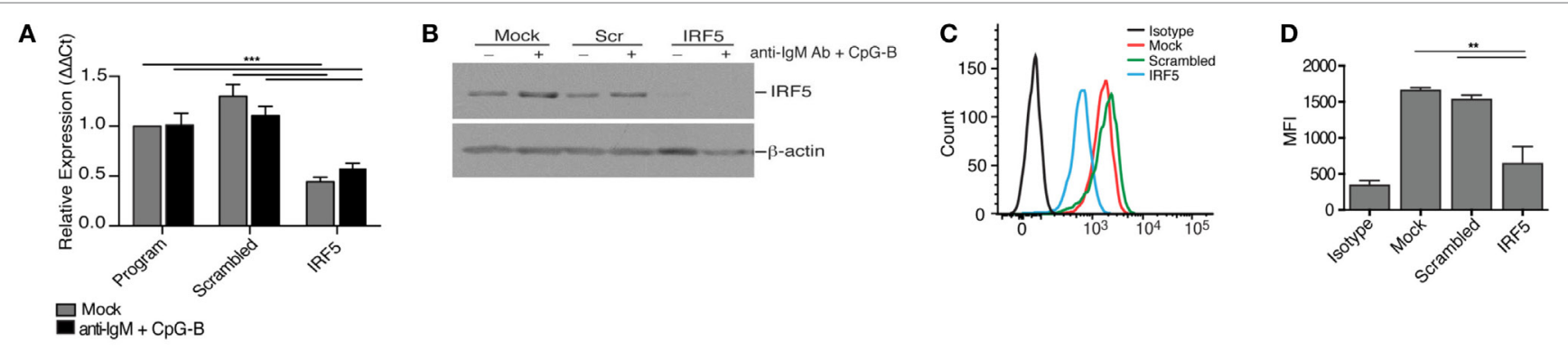

E

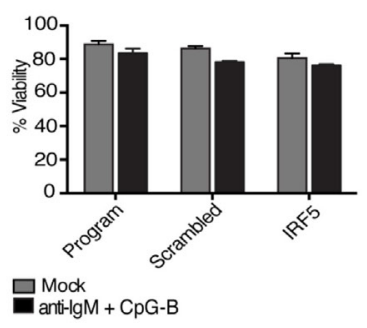

F

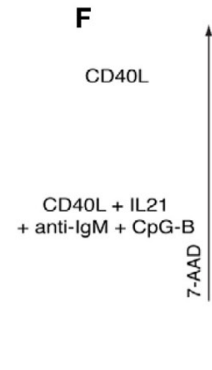

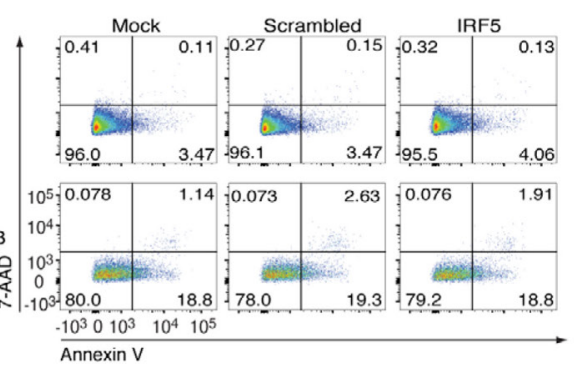

I

H

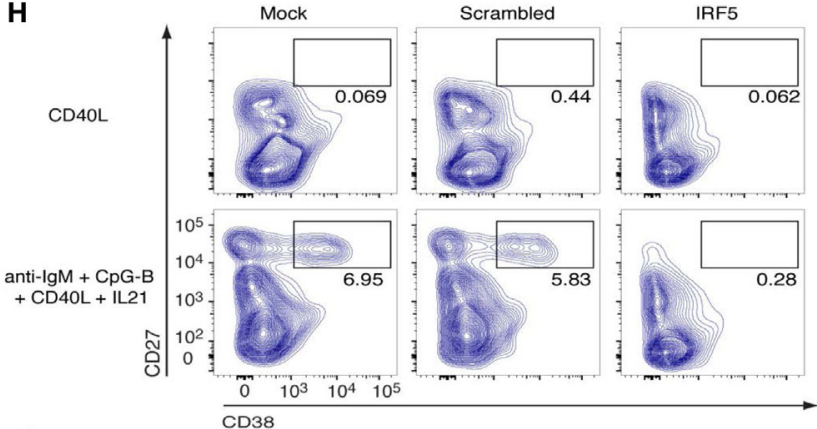

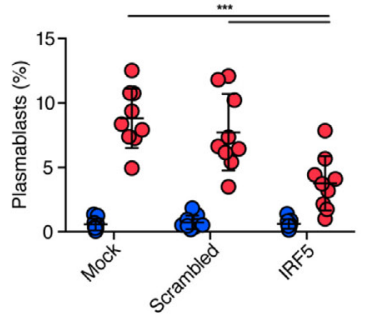

G

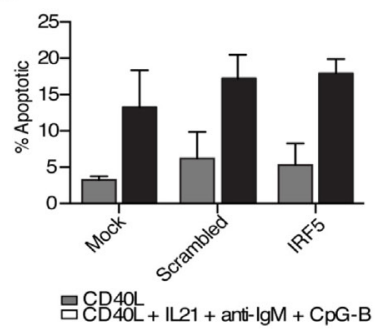

J

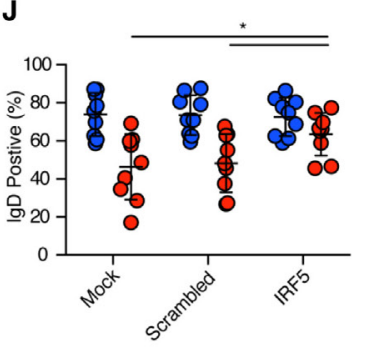

FIGURE 2 | Interferon regulatory factor 5 (IRF5) is required for toll-like receptor 9/B cell receptor-induced antibody secreting cell differentiation. Isolated human naive B cells were nucleofected with $500 \mathrm{nM}$ of mock, scrambled or IRF5 siRNA and mock-stimulated or stimulated with the indicated cocktails. (A) IRF5 transcript expression was quantified through qPCR on RNA isolated 48 h postnucleofection and 12 h poststimulation with anti-lgM+ CpG-B (two-way ANOVA with Tukey's post hoc test; $n=4$ independent donors). (B) Protein lysates were prepared from nucleofected B cells 72 h postnucleofection and 24 h poststimulation with anti-IgM+ CpG-B. Western blot is one representative experiment out of three performed on $n=3$ independent donors. (C) Representative histograms of IRF5 expression $48 \mathrm{~h}$ postnucleofection. Viable cells were analyzed through live/dead staining discrimination. (D) Plotted MFI of IRF5 from (C) (one-way ANOVA with Tukey's post hoc test; $n=4$ independent donors). (E) Plotted percentage of B cell viability assessed $72 \mathrm{~h}$ post-nucleofection, as determined through trypan blue exclusion. Data are from $n=3$ independent donors. (F) Representative dot plots from B cell apoptosis quantified following staining with Annexin $V$ and 7 aminoactinomycin D (7-AAD). Early apoptotic events are characterized as Annexin $\mathrm{V}^{+}$7AAD-, whereas late apoptotic events are Annexin $\mathrm{V}^{+} 7 A \mathrm{AD}^{+}$. Quantitation is shown in (G). (G) Average apoptotic B cells from (F) $96 \mathrm{~h}$ post-nucleofection and $48 \mathrm{~h}$ post-stimulation (Two-way ANOVA with Tukey's post hoc test; $n=3$ independent donors). (H) Isolated naive B cells were nucleofected with $500 \mathrm{nM}$ of mock, scrambled or IRF5 siRNA and stimulated with either CD40L or the combination of CD4OL, IL21, anti-IgM, and CpG-B for 7 days. Plasmablast differentiation was quantified through gating of CD19+CD20+IgD-CD27+CD38+ B cells; final $\mathrm{CD} 27^{+} \mathrm{CD} 38^{+}$gating is shown. Flow cytometry contour plots are representative of one experiment from a single donor. (I) Average number of plasmablasts from (H) following culture for 7 days (two-way ANOVA with Tukey's post hoc test; $n=9$ independent donors). (J) Average number of lgD+CD38lo B cells from (H) following stimulation (two-way ANOVA with Tukey's post hoc test; $n=9$ independent donors). Error bars represent SD. ${ }^{*} p \leq 0.05$; ${ }^{* *} p \leq 0.01$; ${ }^{\star * \star} p \leq 0.001$.

knockdown (Figure 2E). As viability assays often fail to detect early stages of apoptosis, we assayed B cell apoptosis following IRF5 knockdown through Annexin V and 7-amino-actinomycin $\mathrm{D}$ (7-AAD) staining. CD40L treatment did not induce significant levels of apoptosis, whereas stimulation with CD40L, IL21, antiIgM, and CpG-B induced a similar increase in apoptosis across all three siRNA conditions (Figures 2F,G). Upon exposure to antigen, only a small proportion of naive $B$ cells will be selected to undergo ASC differentiation, thus the observed increase in apoptosis by the combination treatment is not surprising. However,
IRF5 knockdown did not result in a significant decrease in apoptosis in comparison to control conditions (Figure 2G). This indicates that under the current experimental conditions, IRF5 does not have a significant impact on B cell viability or apoptosis.

Following stimulation, B cells rapidly proliferate and differentiate to ASCs. In an effort to measure ASC generation from the population of naive B cells showing IRF5 knockdown, naive B cells were conucleofected with GFP mRNA and IRF5 siRNA. Unfortunately, only $\sim 20 \%$ of naive B cells expressed GFP and no correlation between GFP and IRF5 knockdown was found 
(Figures S2A-C in Supplementary Material). Similar effects were seen with pmaxGFPTM; in this case, only $\sim 2-4 \%$ of naive B cells expressed GFP (Figures S2D,E in Supplementary Material). Thus, cells were nucleofected as before and cultured for 2 days, followed by stimulation with CD40L or anti-IgM, CpG-B, CD40L, and IL21 for 7 days. These stimulation conditions result in a significant increase in $\mathrm{IgD}^{-} \mathrm{CD} 27^{+} \mathrm{CD} 38^{+}$plasmablasts $(6,58)$, as seen in both mock and scrambled control (Figures 2H,I, flow gating in Figure S3A in Supplementary Material). In contrast to these controls, IRF5 knockdown resulted in a two-fold reduction in plasmablast differentiation. Additionally, the percentage of $\mathrm{IgD}^{+}$ $B$ cells present following stimulation was significantly increased after IRF5 knockdown (Figure 2J; Figure S3B in Supplementary Material). Similar to that observed in the circulation of influenzavaccinated healthy donors (Figures 1E,F), increased IRF5 expression was detected in B cells over the 7 -day in vitro culture period. As expected, naive B cells expressed the lowest levels of IRF5 and plasmablasts expressed the highest (Figures S2F,G in Supplementary Material). Together, these data indicate that IRF5 has a B cell-intrinsic role in human ASC differentiation.

\section{IRF5 Knockdown Impairs B Cell Proliferation and Activation}

Stimulation of B cells results in a rapid proliferative burst, which is required for sufficient numbers of antigen-specific B cells to survive CSR. To investigate if a decrease in the number of $\mathrm{B}$ cells entering proliferation is responsible for the reduction in plasmablasts seen after IRF5 knockdown, we assayed B cell proliferation. Nucleofected B cells were labeled with the proliferation dye CFSE, stimulated with anti-IgM, CpG-B, CD40L, and IL21 for 5 days, and CFSE dilution determined through flow cytometery by gating on viable cells (Figure S3A in Supplementary Material). A significant reduction in the number of naive B cells undergoing proliferation was seen following IRF5 knockdown (Figures 3A,B). Compared to mock and scrambled controls, IRF5 knockdown resulted in a twofold reduction in proliferation.

B cell activation occurs immediately following antigen recognition, and can occur through each of the previously described activation pathways. CpG-B stimulation of naive $\mathrm{B}$ cells can upregulate expression of the activation marker CD86 (6). As B cell activation primes for proliferation, we assayed activation following IRF5 knockdown. Primary naive B cells were nucleofected and stimulated for 2 days. Stimulation induced the upregulation of CD86 expression in both mock- and scramble-nucleofected B cells, whereas IRF5 knockdown significantly reduced CD86 expression (Figure 3C). IRF5 is known to function downstream of TLR9, which has previously been shown to be involved in the upregulation of CD86 $(52,58)$. These data indicate that IRF5 acts downstream of TLR9/BCR to induce early B cell activation and proliferation.

\section{IRF5 Knockdown Reduces Human IgG1 and 3 Secretion}

Given the reduction in plasmablast differentiation seen after IRF5 knockdown, we determined whether there would be a consequential reduction in secreted antibody. We first measured percentages of nucleofected B cells expressing intracellular IgA, IgE, or IgG following stimulation for 5 days using flow cytometry. Intracellular Ig staining is indicative of the fraction of B cells that may secrete a specific antibody isotype. Representative flow gating for IgG in total $\mathrm{CD} 19^{+} \mathrm{CD} 20^{+} \mathrm{B}$ cells is shown in Figure 3D. While few B cells expressed intracellular IgA or IgE, a large fraction of B cells stained positive for IgG. IRF5 knockdown resulted in a significant reduction in intracellular IgG staining (Figure 3E). IRF5 has previously been linked to IgG isotype secretion in mice, with $\operatorname{Irf5^{-/}}$ mice having reduced IgG2a levels following viral infection and in murine models of lupus $(21,22)$. As IL21 and CpG have both been shown to predominantly promote IgG secretion (59), we further measured IgG isotypes by ELISA. Following stimulation, IRF5 knockdown resulted in significantly decreased levels of IgG1, 2, and 3 but not 4 (Figure 3F). Since IgG2 and 4 were only secreted to a low extent, the largest impact was among the IgG1 and 3 isotypes. To determine if the reduction in IgG expression was due to a reduction in CSR, we assayed AID expression. AID expression is transcriptionally regulated, with expression quickly upregulated following B cell activation and proliferation. Interestingly, expression of AID was significantly decreased following IRF5 knockdown (Figure 3G). These data indicate that IRF5 contributes to early stages of ASC differentiation.

\section{Identification of New IRF5 Target Genes Associated with ASC Differentiation}

Interferon regulatory factor 5 initiates transcription of target genes following nuclear translocation; however, IRF5 target genes in B cells have not been characterized. We performed chromatin IP combined with deep sequencing (ChIP-Seq) to identify IRF5 transcriptional targets across the genome in human primary naive B cells. To ensure IRF5 is specifically enriched in ChIP experiments, previously validated antibodies were used for IP (36). ChIP-Seq libraries were prepared from both mock and anti-IgM ${ }^{+} \mathrm{CpG}-\mathrm{B}$-stimulated cells $(4 \mathrm{~h})$ to characterize activation-dependent targets of IRF5. Computational analysis revealed a sharp increase in the number of IRF5 peaks following stimulation. In mock-treated samples, IRF5 bound 22 genes, and upon treatment with anti-IgM+ $\mathrm{IpG}^{+} \mathrm{B}$, IRF5 targets increased to 784. IRF5 genome occupancy was distributed across various gene elements with $3.5 \%$ of peaks occurring within $\pm 3 \mathrm{~kb}$ of putative transcription start sites (Figure 4A). Additionally, a large majority of peaks were found on intragenic regions. Roughly $10.5 \%$ of peaks were bound to exons, and $21 \%$ were bound to non-coding regions.

Interferon regulatory factor family members bind to IFN-stimulated response elements (ISREs) consisting of the GAANNGAA motif. IRF5, however, can partner with other transcription factors to recognize various consensus sequences. It was recently reported that IRF5, in cooperation with RelA of the NFkB pathway, can recognize composite PU.1/ISRE motifs in murine inflammatory monocytes (60). IRF5 consensus sites have not been well-defined in humans. We therefore sought to determine if IRF5 recognizes similar motifs in human B cells. 


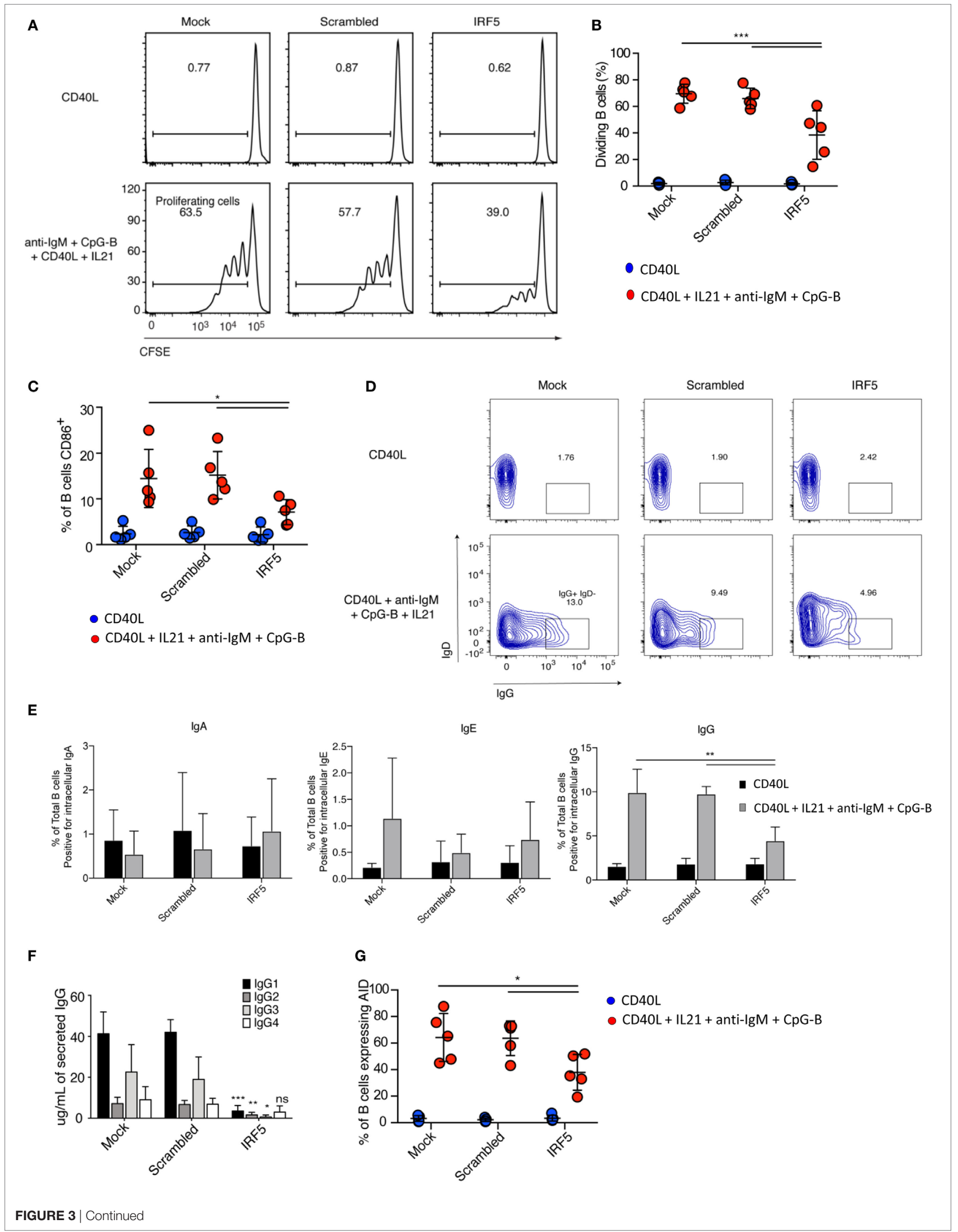


FIGURE 3 | Interferon regulatory factor 5 (IRF5) knockdown impairs B cell proliferation, activation, and immunoglobulin (Ig) G isotype secretion. (A) Representative flow cytometry histograms from cell proliferation assay as measured through dilution of the proliferation dye CFSE. Isolated primary naive B cells from a single donor were nucleofected with $500 \mathrm{nM}$ of mock, scrambled or IRF5 siRNA and stimulated with either CD40 ligand (CD4OL) or the combination of CD40L, IL21, anti-IgM, and CpG-B for 5 days. (B) Average percentage of proliferating B cells from (A) is shown (two-way ANOVA with Tukey's post hoc test; $n=5$ independent donors). (C) Average percentage of B cells expressing CD86. Similar to (A) except isolated naive B cells were nucleofected and then stimulated with CD40L, IL21, anti-IgM, and CpG-B for $24 \mathrm{~h}$, then stained for surface CD86 (two-way ANOVA with Tukey's post hoc test; $n=5$ independent donors). (D) Representative contour plots from IgD-lgG $\mathrm{B}$ cells after gating on total $\mathrm{CD} 19^{+} \mathrm{CD}_{20} \mathrm{O}^{+} \mathrm{B}$ cells. Isolated primary naive B cells were nucleofected with $500 \mathrm{nM}$ of mock, scrambled or IRF5 siRNA and stimulated with either CD4OL or the combination of CD4OL, IL21, anti-IgM, and CpG-B for 7 days. (E) Quantification from (D) of Ig antibody class expression was determined by intracellular flow cytometery (one-way ANOVA with Tukey's post hoc test; $n=5$ independent donors). (F) Cell culture supernatants from (D) were used for ELISA to determine IgG isotype secretion. Average concentration of IgG isotype is shown (two-way ANOVA with Tukey's post hoc test; $n=4$ independent donors). (G) Frequency of activation-induced deaminase (AID) expression in B cells following nucleofection with $500 \mathrm{nM}$ of mock, scrambled or IRF5 siRNA and stimulated with CD4OL, IL21, anti-IgM, and CpG-B for 3 days. AID expression was determined by intracellular flow cytometry (one-way ANOVA with Tukey's post hoc test; $n=5$ independent donors). Error bars represent SD. ${ }^{*} p \leq 0.05 ;{ }^{* \star} p \leq 0.01 ;{ }^{* \star *} p \leq 0.001$.

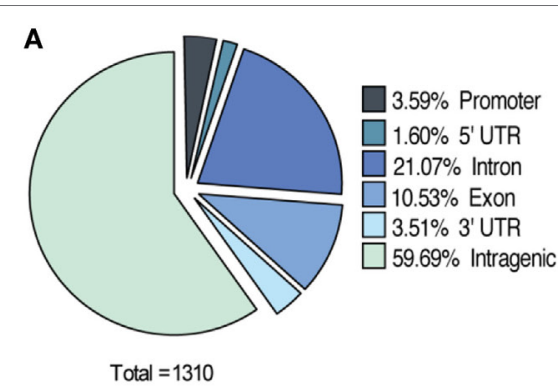

B

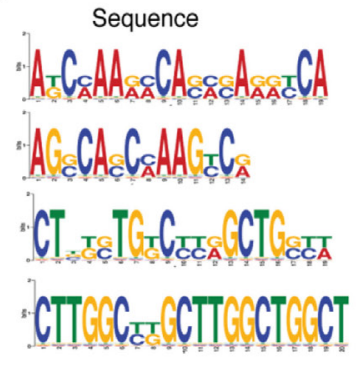

Motif

IRF5/RUNX

PAX5

CTCF

NKF1
C
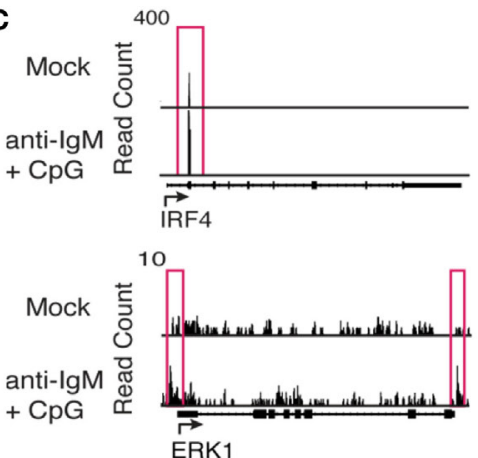

100

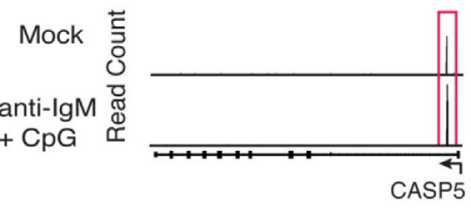

20

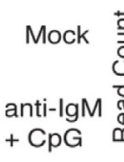

$+\mathrm{CpG}$

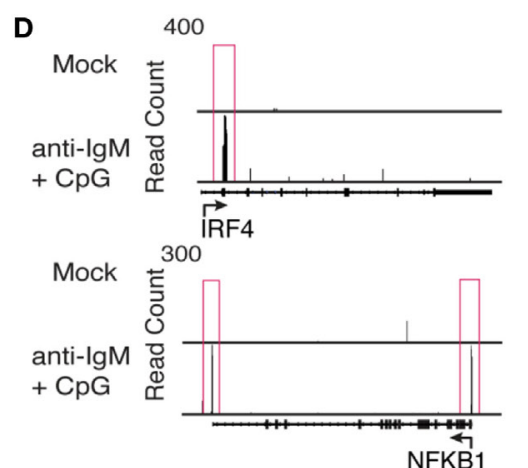

NFKB1
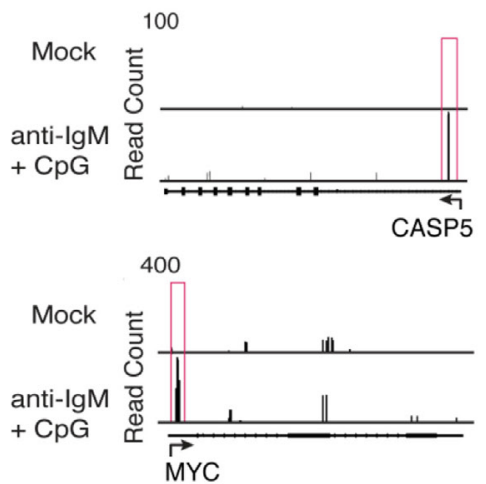

E

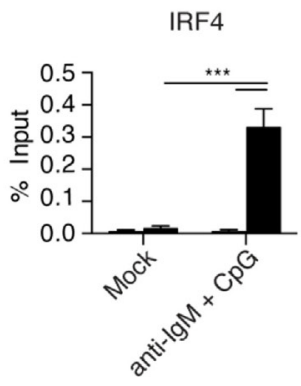

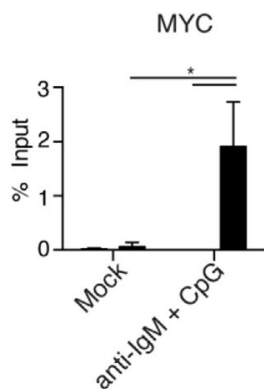

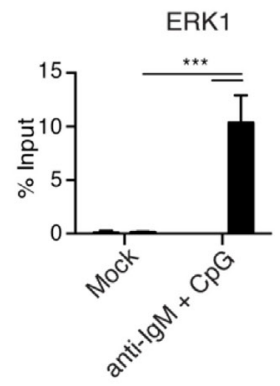

FIGURE 4 | Interferon regulatory factor 5 (IRF5) binds promoter regions of genes associated with antibody secreting cell (ASC) differentiation. IRF5 ChIP-Seq was performed on isolated primary naive B cells from $n=2$ independent donors. B cells were either mock or anti-lgM+ CpG-B stimulated for $4 \mathrm{~h}$. Reads were mapped through BWA and peaks called through MACs. (A) A pie chart showing representative IRF5 binding elements throughout the human primary B cell genome.

(B) Common IRF5 binding motifs identified from ChIP-Seq. Motif sequences are shown in order of enrichment with associated transcription factor motif.

(C) Representative peak distributions are shown for IRF4, ERK1, CASP5, and MYC. Peaks are boxed in red and were determined at a significance of $p \leq 0.0001$.

(D) Same as (C) except IRF5 ChIP-Seq peaks are from the Ramos B cell line. Peaks were determined at $p \leq 10^{-10}$. (E) Independent confirmation of IRF5 binding to IRF4, MYC, and ERK1 target sites through ChIP-qPCR in primary naive B cells mock stimulated or stimulated with anti-lgM+ $\mathrm{CpG}-\mathrm{B}$ for $4 \mathrm{~h}$ (two-way ANOVA with Tukey's post hoc test; $n=4$ independent donors). Error bars represent SD. ${ }^{*} p \leq 0.05 ;{ }^{\star \star \star} p \leq 0.001$. 

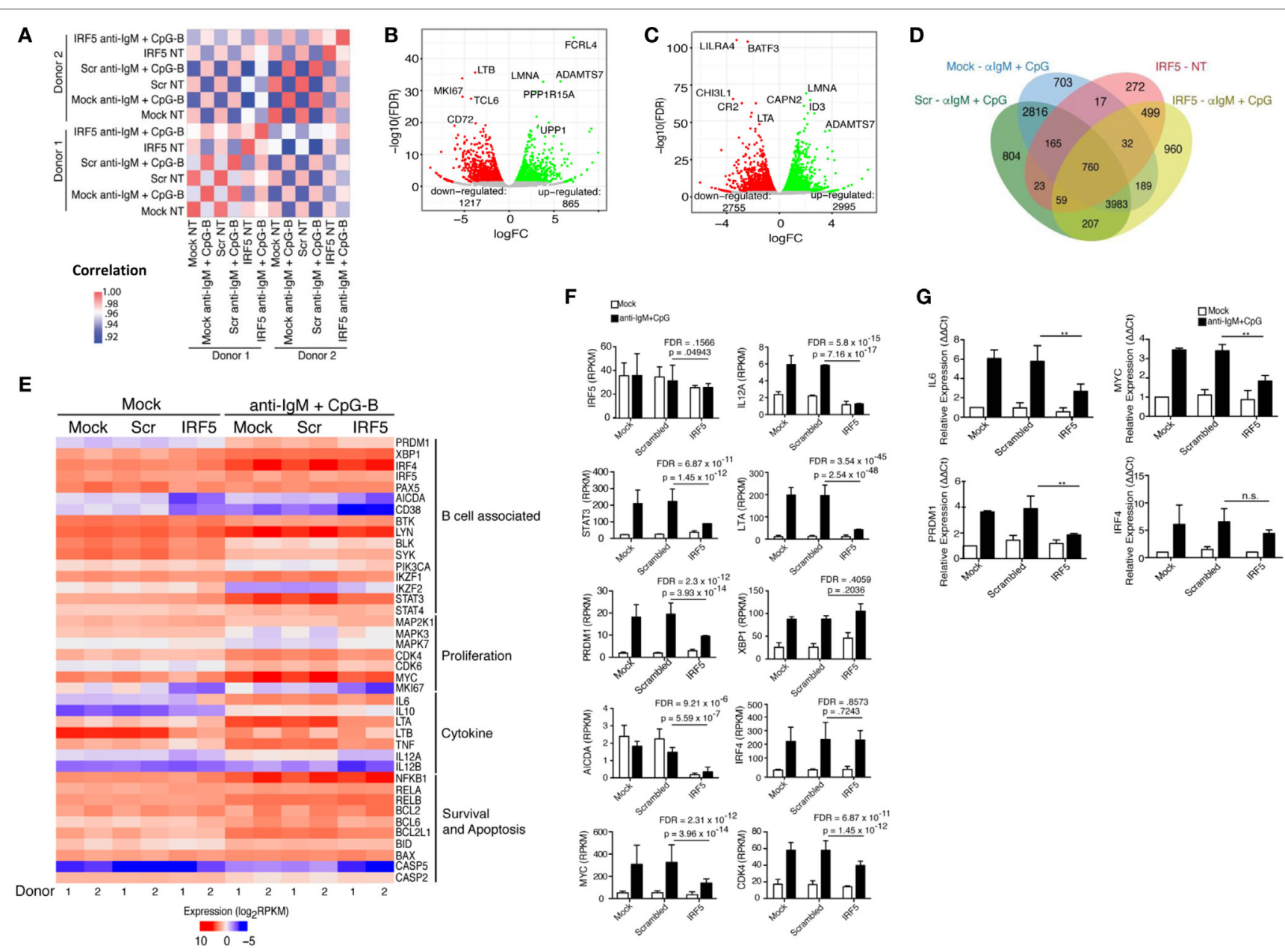

F anock
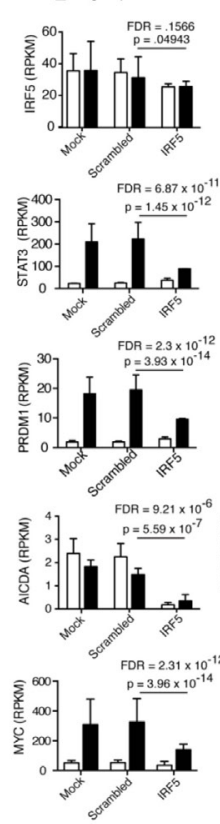
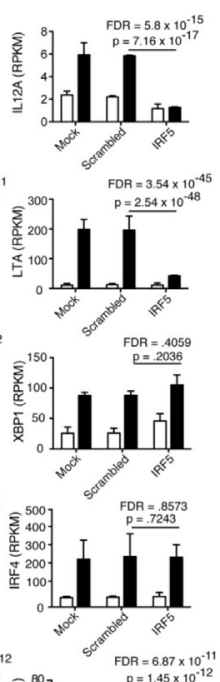

G

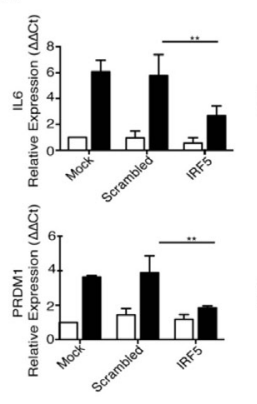

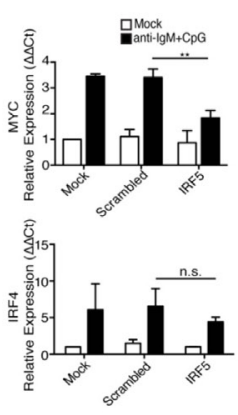

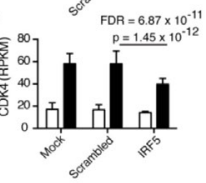

FIGURE 5 | Identification of an interferon regulatory factor 5 (IRF5)-dependent B cell transcriptome. Isolated naive B cells were nucleofected with 500 nM of mock, scrambled or IRF5 siRNA and subsequently stimulated with anti-IgM and CpG for $6 \mathrm{~h}$. RNA-seq was performed on B cells from $n=2$ independent donors.

(A) Significant donor correlation associated with IRF5 knockdown and stimulation. Heat map illustrates correlation coefficients for gene expression values between conditions (nucleofection and stimulation) and individual healthy donors. A strong correlation between samples is indicated by the red and pink boxes. Color legend is shown to the right indicating correlation coefficients. (B) Identification of genes with differential expression following IRF5 knockdown in mock-treated samples. Red circles indicate downregulated genes; green circles indicate upregulated genes. Differential gene expression was determined in comparison to scrambled control. (C) Same as (B) except genes were identified following IRF5 knockdown and stimulation (CpG-B+ anti-lgM). Differential expression was determined by comparison to stimulated scrambled control. (D) Diagram of differential expression between nucleofection and various stimulation conditions. Overlapping regions represent shared expression; single regions represent uniquely expressed genes. (E) Heat map of gene expression between the two donors based on cellular function associated with B cell activation, proliferation, and antibody secreting cell differentiation. Data are expressed as log 2 RPKM. (F) Raw RPKM values of particular genes relevant to IRF5 function. $p$ values and false discovery rate (FDR) scores were obtained by multiple comparisons testing of samples indicated by line. Individual $p$ values and FDR scores are included in each graph. (G) Independent confirmation of differential gene expression through qPCR (two-way ANOVA with Tukey's post hoc test; $n=3$ independent donors). Error bars represent SD. ${ }^{* \star} p \leq 0.01$.

Using motif discovery algorithms, we identified common motifs within $100 \mathrm{bp}$ of peak centers. Among the represented motifs was the expected ISRE consensus sequence, as well as a newly identified IRF5/RUNX motif that was equally enriched for (Figure 4B). These data suggest cooperation between IRF5 and RUNX family members in the targeting of genes following B cell activation.

Interferon regulatory factor 5 was found to bind a wide range of genes, including several associated directly with ASC differentiation. Among these targets were IRF4, ERK1, and CASP5 (Figure 4C). While these genes are implicated in ASC differentiation and were targeted by IRF5 in human primary B cells following activation, none seemed to fully account for the early defects in B cell proliferation or activation (Figures $\mathbf{3 A - C}$ ). As a result, there remained the possibility that several target genes were missing due to limitations, possibly, in obtaining sufficient numbers of primary naive B cells at the $4 \mathrm{~h}$ time point of IRF5 activation (Figure 1C). To ensure the identification of all possible IRF5 target genes, we performed IRF5 ChIP-Seq in Ramos B cells. Ramos are known to express high levels of TLR9, and thus recapitulate early stages of primary naive $B$ cell activation following anti-IgM ${ }^{+} \mathrm{CpG}-\mathrm{B}$ stimulation (61). Enriched reads from Ramos B cells were similarly distributed as in primary 
TABLE 1 | List of top differentially expressed genes comparing mock-treated with scrambled siRNA against IRF5 knockdown. ${ }^{a}$

\begin{tabular}{|c|c|}
\hline \multicolumn{2}{|c|}{ Upregulated genes in mock-treated cells } \\
\hline Gene $^{b}$ & Function \\
\hline FCRL4 & $\begin{array}{l}\text { Member of the Ig receptor superfamily; acts as an inhibitor } \\
\text { of B cell activation }\end{array}$ \\
\hline ADAMTS7 & $\begin{array}{l}\text { Plays a role in activation of vascular smooth muscle cells. } \\
\text { Unknown role in B cells }\end{array}$ \\
\hline LMNA & $\begin{array}{l}\text { Lamin A/C, major component of the nuclear lamina. Important } \\
\text { in maintaining integrity of nuclear structure; involved in } \\
\text { chromosome segregation during mitosis }\end{array}$ \\
\hline PPP1R15A & $\begin{array}{l}\text { Recruits protein phosphatase 1, which suppresses stress } \\
\text { kinase-induced protein expression. Expression upregulated } \\
\text { during cellular stress or DNA damage }\end{array}$ \\
\hline AMICA1 & $\begin{array}{l}\text { Adhesion molecule expressed on cell surface; primarily } \\
\text { on T cells. Function in B cells remains unclear }\end{array}$ \\
\hline CD109 & $\begin{array}{l}\text { A glycosyl phosphatidylinositol (GPI)-linked glycoprotein that } \\
\text { localizes to the cell surface. Acts as a TGF-b coreceptor which } \\
\text { stimulates internalization and degradation of TFG-b receptors }\end{array}$ \\
\hline UPP1 & $\begin{array}{l}\text { Catalyzes cleavage of uridine and deoxy-uridine to uracil and } \\
\text { ribose- or deoxyribose-1-phosphate }\end{array}$ \\
\hline SLC3A2 & $\begin{array}{l}\text { Cell surface transmembrane protein which makes up the heavy } \\
\text { chain of the amino acid transporter CD98. Acts as an integrin } \\
\text { binding protein. Shown to be required for B cell proliferation and } \\
\text { antibody responses }\end{array}$ \\
\hline SQSTM1 & $\begin{array}{l}\text { Sequestosome 1, a multifunctional signaling protein known to } \\
\text { be an autophagy target as well as an ubiquitin binding scaffolding } \\
\text { protein involved in NFkB activation through interaction with TRAF6 }\end{array}$ \\
\hline \multicolumn{2}{|c|}{ Downregulated genes in mock-treated cells } \\
\hline$\angle T B$ & $\begin{array}{l}\text { Lymphotoxin beta, membrane protein of the TNF family. } \\
\text { Inflammatory B cell cytokine }\end{array}$ \\
\hline CHI3L1 & $\begin{array}{l}\text { Member of chitanase family of proteins; lacks chitanse activity. } \\
\text { Unclear role in B cells }\end{array}$ \\
\hline MKI67 & $\begin{array}{l}\text { Ki67, surfactant required for chromosome segregation in } \\
\text { proliferating cells. Used as marker of proliferating cells }\end{array}$ \\
\hline TCL6 & Non-coding RNA of unknown function \\
\hline CPNE5 & $\begin{array}{l}\text { Copine } 5 \text {, calcium binding protein involved in regulating } \\
\text { cell signaling }\end{array}$ \\
\hline TRIB2 & $\begin{array}{l}\text { Member of tribble family of serine/threonine kinases. } \\
\text { Regulates TLR5-mediated NFkB activation }\end{array}$ \\
\hline CD72 & $\begin{array}{l}\text { Membrane protein containing ITIM motifs and C-type lectin } \\
\text { domains. Negative regulator of BCR-mediated signaling by } \\
\text { suppression of calcium mobilization. Recently shown to regulate } \\
\text { B cell autoreactivity following TLR7 ligation with autoantigen Sm/ } \\
\text { RNP }\end{array}$ \\
\hline CORO2B & Coronin 2B protein coding gene of unknown function \\
\hline FAM129C & B cell novel protein 1 , unknown signaling protein \\
\hline
\end{tabular}

${ }^{a}$ Comparing gene expression between scrambled siRNA and IRF5 siRNA nucleofected samples.

${ }^{b} p$-Value $\leq 0.05$ and an FDR $<0.05$ after Benjamin-Hochberg correction was used for determining significant differential gene expression. Genes are from Figure $\mathbf{5}$.

B cells (Figure S4A in Supplementary Material). Importantly, identical target genes, such as IRF4 and CASP5, were identified in both datasets (Figures 4C,D). Several additional target genes not seen in primary B cells were identified in Ramos. Among
TABLE 2 | List of top differentially expressed genes comparing anti-IgM+ $\mathrm{CpG}-\mathrm{B}$ stimulated with scrambled siRNA against IRF5 knockdown. ${ }^{a}$

\section{Upregulated genes in anti-IgM+ CpG-B-treated cells}

NR4A1 Hormone nuclear receptor and transcription factor. Involved in cell cycle progression and regulation of apoptosis. NR4A1(Nur77)deficient mice display increased $\mathrm{B}$ cell survival among $\mathrm{CD}^{+}{ }^{+}$ B cells. Increased autoantibody production also observed

\section{LMNA See above}

ID3 Transcriptional regulator of basic helix-loop-helix family of transcription factors. Implicated in cell growth, apoptosis, and differentiation. Required for germinal center formation but acts as a negative regulator of plasma cell differentiation

LPXN Leupaxin, a negative regulator of paxilin signaling. Negative regulator of BCR signaling

NR4A2 Hormone nuclear receptor and transcription factor

CAPN2 Calpain 2, an intracellular cysteine protease. Regulates cell migration; is a Pax5 target gene in B cells

ADAMTS7 See above

ARL4C Small GTP binding protein and member of ADP-ribosylation factor family. Regulates microtubule dependent intracellular vesicular transport

FLNA Filamin A, an actin binding protein responsible for cross-linking of actin filaments. Plays a role in regulating cell shape and structure

\section{Downregulated genes in anti-IgM+ CpG-B-treated cells}

LILRA4 Ig-like receptor expressed on cell surface. Receptor for bone marrow stromal cell antigen-2; may be involved in regulating early B cell development

BATF3 Basic Leucine Zipper transcription factor within the AP-1 family of transcription factors. Interacts with Jun as a transcriptional regulator in several immune cell populations

CHI3L1 See above

HAPLN3 Member of hyaluronan and proteoglycan binding link protein gene family; major component of extracelluar matrix. Recently shown to be upregulated in IFNb-stimulated B cells

MYBL2 Member of MYB transcription factor family; regulates cyclin D1 expression. Regulates cell cycle progression. Highly expressed during early B cell development

GCA Grancalcin, a calcium binding protein that interacts with TLR9 to mediate IRF7- and NFkB-dependent type 1 interferon expression

SLC2A5 Acts as a fructose transporter. Unclear role in B cells

CR2 Complement receptor 2; binds complement factor C3Dd. Involved in B cell activation

LTA Lymphotoxin alpha; member of TNF family of cytokines. Mediates inflammatory and cytotoxic effects on various target cells

${ }^{a}$ Comparing gene expression between scrambled siRNA and IRF5 siRNA nucleofected samples.

${ }^{b} p$-Value $\leq 0.05$ and an FDR $<0.05$ after Benjamin-Hochberg correction was used for determining significant differential gene expression. Genes are from Figure $\mathbf{5}$.

these was the proliferation gene MYC, and NFKB1 (Figure 4D). To confirm that results from Ramos B cells were applicable to primary B cells, IRF5 ChIP-qPCR was performed on pooled primary naive B cells. Significant enrichment was seen on IRF4, $M Y C$, and ERK1 following IRF5 ChIP (Figure 4E). Interestingly, $M Y C$ is downstream of ERK1 and an ERK1-Elk-Myc signaling pathway has been implicated in early B cell proliferation $(8,62)$. 
TABLE 3 | List of ASC-associated genes from RNA-Seq that contain potential ISRE and/or IRF-E sites in their regulatory regions. ${ }^{\text {a }}$

\begin{tabular}{|c|c|c|c|}
\hline Gene & Chromosome position (Hg19) & ISRE & IRF-E \\
\hline PRDM1 & chr6:106,534,195-106,557,814 & $\begin{array}{l}106566840 \\
106547222\end{array}$ & 106562388 \\
\hline XBP1 & chr22:29,190,548-29,196,560 & ND & ND \\
\hline IRF4 & chr6:391,739-411,443 & 391724 & 409414 \\
\hline IRF5 & chr7:128,577,994-128,590,088 & 128580725 & 128594612 \\
\hline PAX5 & chr9:36,838,531-37,034,476 & ND & $\begin{array}{l}36867441, \\
36868076, \\
37197361\end{array}$ \\
\hline$A / C D A$ & chr12:8,754,762-8,765,442 & ND & ND \\
\hline CD38 & chr4:15,779,931-15,850,706 & ND & ND \\
\hline BTK & chrX:100,604,435-100,641,212 & ND & 100668558 \\
\hline$L Y N$ & chr8:56,792,386-56,925,006 & $\begin{array}{l}56842848 \\
56863323\end{array}$ & $\begin{array}{l}6773360 \\
56793604\end{array}$ \\
\hline$B L K$ & chr8:11,351,521-11,422,108 & ND & 11349703 \\
\hline IKZF1 & chr7:50,344,378-50,472,798 & $\begin{array}{l}50340355 \\
50484586\end{array}$ & $\begin{array}{l}50424402, \\
50444411 \\
50484587\end{array}$ \\
\hline$I K Z F 2$ & chr2:213,864,411-214,015,058 & $\begin{array}{l}213831888, \\
214030738, \\
214014429, \\
214017464, \\
214030738\end{array}$ & $\begin{array}{l}213936673, \\
214015411, \\
214017463\end{array}$ \\
\hline STAT3 & chr17:40,465,343-40,540,513 & 40460528 & ND \\
\hline STAT4 & chr2:191,894,302-192,016,322 & ND & $\begin{array}{l}191885173 \\
191964289\end{array}$ \\
\hline$M Y C$ & chr8:128,748,315-128,753,680 & ND & ND \\
\hline MKI67 & chr10:129,894,925-129,924,468 & ND & ND \\
\hline IL6 & chr7:22,766,766-22,771,621 & ND & $\begin{array}{l}22763062 \\
22765259\end{array}$ \\
\hline IL12A & chr3:159,706,623-159,713,806 & ND & ND \\
\hline NFKB1 & chr4:103,422,486-103,538,459 & $\begin{array}{l}103424172 \\
103560981\end{array}$ & $\begin{array}{c}103448890 \\
103459016, \\
103533964\end{array}$ \\
\hline$R E L A$ & chr11:65,421,067-65,430,443 & ND & 65431520 \\
\hline$R E L B$ & chr19:45,504,707-45,541,456 & ND & ND \\
\hline CASP5 & chr11:104,864,967-104,893,895 & ND & ND \\
\hline
\end{tabular}

${ }^{a}$ Consensus sequences were identified through UCSC using $\mathrm{Hg} 19$ and transcription factor binding sites that are within $4 \mathrm{~kb}$ upstream of the transcription start site (TSS) and $4 \mathrm{~kb}$ downstream of the stop codon.

ND, not detected.

These data support that IRF5 directly binds to promoters of genes important for early stages of B cell activation and proliferation.

\section{Identification of an IRF5-Dependent B Cell Transcriptome}

Identification of IRF5 target genes suggested that IRF5 could influence the transcription of several genes important for ASC differentiation. We thus examined changes on the B cell transcriptome following IRF5 knockdown by high throughput cDNA sequencing (RNA-Seq). Nucleofected B cells were mock or anti-IgM+ $\mathrm{CpG}-\mathrm{B}$ stimulated for $6 \mathrm{~h}$; the $6 \mathrm{~h}$ timepoint was chosen to account for early IRF5-mediated gene transcription occurring in response to nuclear IRF5 at $4 \mathrm{~h}$ poststimulation (Figure 1C). RNA-Seq was performed on two independent biological replicates showing a strong correlation coefficient between gene expression (Figure 5A). Bioinformatics analysis found a dramatic shift in the transcriptome of $\mathrm{B}$ cells following knockdown (Figures S4B,C in Supplementary Material). After knockdown in mock-treated cells, 1,217 genes were downgregulated while 865 genes were upregulated (Figure 5B). The most differentially expressed genes following IRF5 knockdown in the mock-treated group are listed in Table $\mathbf{1}$ (and shown in Figure S4B in Supplementary Material). Genes such as FcRL4 and SLC3A2, which were significantly upregulated following IRF5 knockdown, have been shown to regulate B cell activation and proliferation. FCRL4 is known to act as an inhibitor of BCR signaling (63). SLC3A2 was shown to be important for $B$ cell proliferation and plasmablast differentiation in mice (64). Alternatively, the B cell cytokine $L T B$, as well as the proliferation marker Ki67 were found to be significantly downregulated after IRF5 knockdown (65). Following stimulation, IRF5 knockdown resulted in 2,755 downregulated and 2,995 upregulated genes (Figure 5C). Among the most differentially expressed genes within this group (Figure S4C in Supplementary Material; Table 2), which are relevant to $B$ cell activation, included the upregulated genes ID3 and NR4A1 (66-68). Both have been shown to be involved in B cell activation and proliferation. In contrast, the B cell-associated cytokine LTA and the proliferation marker Ki67 were found both to be significantly downregulated.

Differential expression contrasts between stimulated mock-, scrambled-, and IRF5-nucleofected samples identified several genes uniquely modulated following IRF5 knockdown (Figure 5D). While several of these genes showed significant differential expression and have been previously implicated in B cell biology, they failed to intuitively explain the observed reduction in B cell proliferation and differentiation. As a result, we performed a further gene expression analyses focused on genes relevant to $\mathrm{B}$ cell activation, proliferation, and ASC differentiation (Figure 5E). Confirmation of our stimulation conditions was seen through the significant upregulation of genes involved in ASC differentiation (IRF4, XBP1, PRDM1), inflammatory cytokine expression ( $L T A, L T B)$, and proliferation (MYC, CDK4) (Figure 5E). Analysis of differential expression following IRF5 knockdown revealed a significant decrease in many of the genes (Figures 5E,F). As expected, expression of inflammatory cytokines such as IL6, LTA, LTB, and IL12A was significantly reduced following knockdown. Among the genes known to regulate ASC differentiation, expression of PRDM1, $A I C D A$, and $M Y C$ were significantly downregulated whereas expression of IRF4 and XBP1 were not significantly impacted by IRF5 knockdown at this time point (Figure 5F). This may suggest that while IRF5 binds to the IRF4 promoter, it is dispensable in IRF4 transcriptional regulation. In contrast, IRF5 occupancy was found on the promoter region of $M Y C$, and a corresponding significant decrease in $M Y C$ gene expression was seen after knockdown (Figure 5F). Reduction in $M Y C$, and other genes identified by RNA-Seq, was confirmed by real-time qPCR 
in independent donors (Figure 5G). We next examined genes identified in Figure 5E for IRF-E or ISRE consensus binding sites in their 5'- and/or 3'-UTR. Results in Table 3 reveal that while many of the genes do contain consensus binding sites that IRF5 may recognize, not all do. Instead, IRF5 may utilize non-classical sequences, such as those identified in Figure 4B for direct regulation. Altogether, data indicate that one mechanism by which IRF5 regulates human ASC differentiation is through the early control of B cell proliferation via direct regulation of $M Y C$ expression followed by alterations in the expression of other ASC-associated genes.

To gain a further understanding of IRF5-regulated pathways in primary naive $B$ cells, we performed an in-depth integrative analysis of ChIP-Seq and RNA-Seq datasets. Results in Figures 6A,B are from pathway analysis showing the top 20 most activated pathways when compared to mock untreated samples. Data in Figure 6A show a distinct upregulation in the unfolded protein response, adipogenesis, and translation factor pathways after IRF5 knockdown. Conversely, knockdown of IRF5 in untreated and treated samples resulted in the downregulation of cell cycle and BCR signaling pathways (Figure 6B). Downregulation of these pathways coincide with functional defects detected after IRF5 knockdown (Figures 2 and 3). Given that the NF-кB signaling pathway has been shown to play a role in murine ASC differentiation (69), we mapped out genes in this pathway that were affected by IRF5 knockdown (Figure 6C). We also mapped out cell cycle and DNA damage signaling genes affected by IRF5 knockdown since we detected an alteration in BCR/ TLR9-induced cell proliferation (Figure 7). Last, given that early defects in B cell activation were detected after IRF5 knockdown, we mapped out the complex interaction of genes involved in the BCR signaling pathway that were differentially affected (Figure S5 in Supplementary Material). Together, these data implicate IRF5 in the regulation of multiple signaling pathways involved in ASC differentiation and B cell survival.

\section{DISCUSSION}

The differentiation of ASCs is reliant upon a large network of transcription factors, which are responsive to various B cell activation pathways. Among the B cell activation pathways known to play a role in ASC differentiation is the TLR signaling pathway

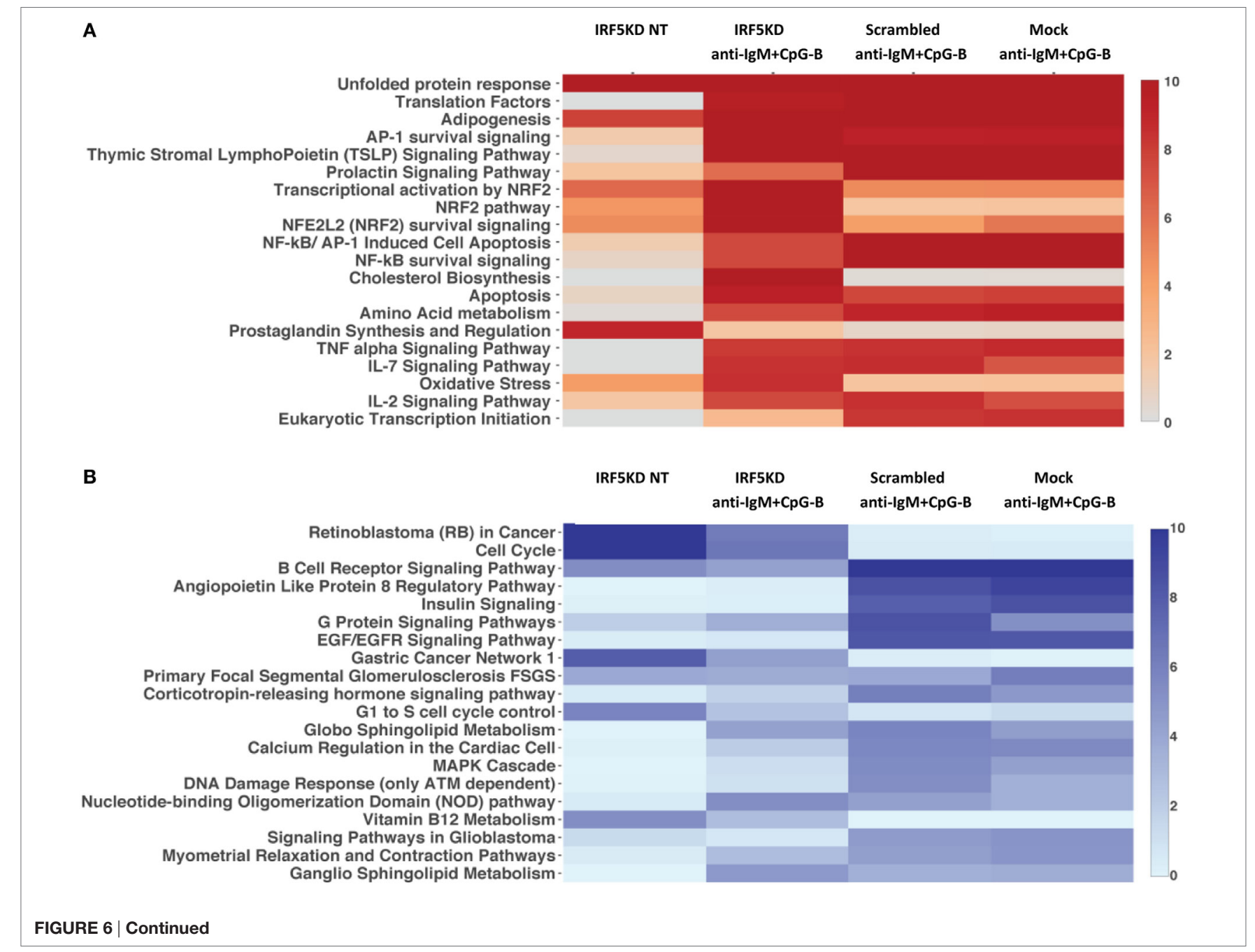


C

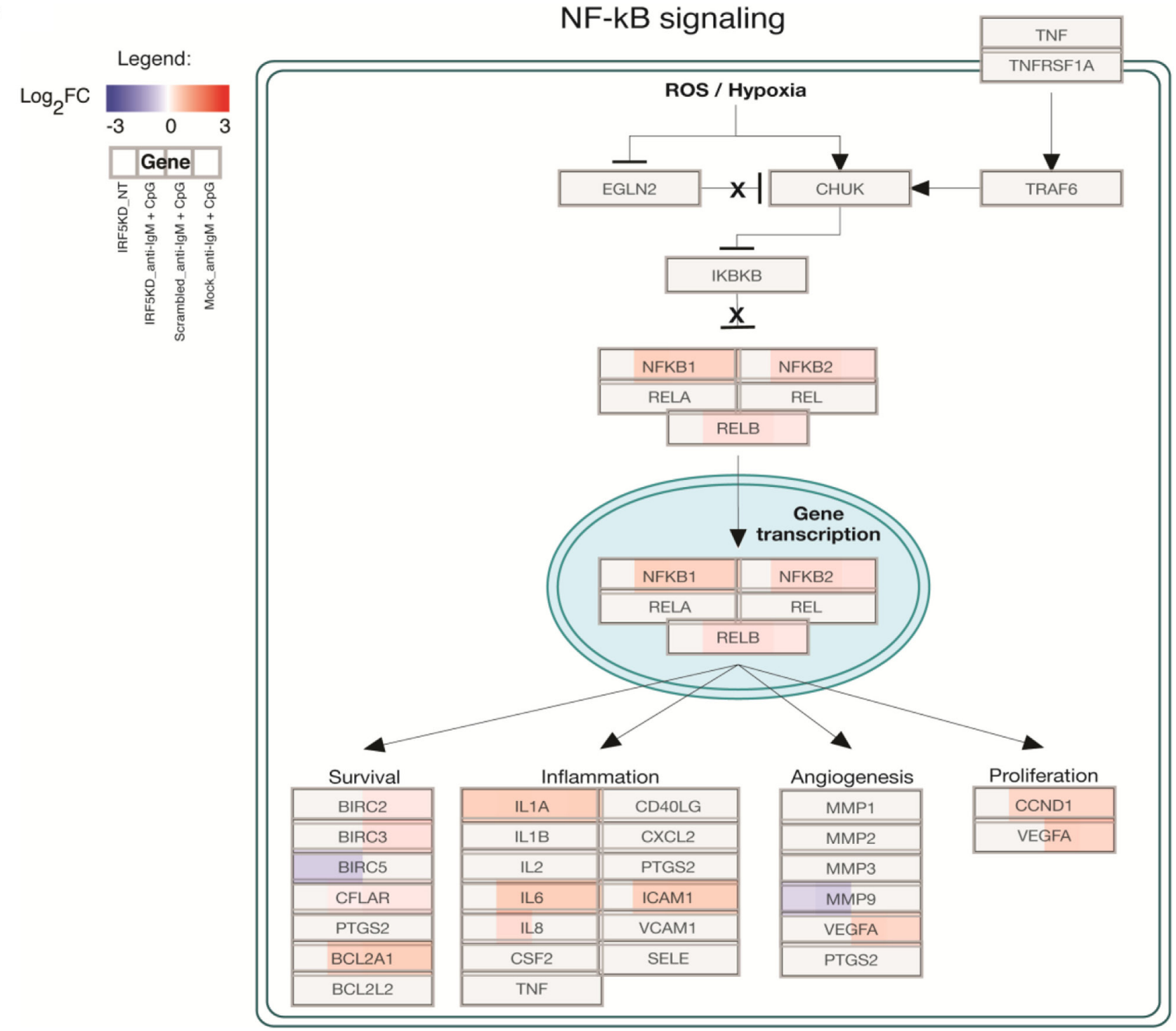

FIGURE 6 | Interferon regulatory factor 5 (IRF5) regulates numerous biologic signaling pathways in human primary B cells. (A) Data from ChIP-Seq and RNA-Seq were used to identify the top 20 upregulated pathways in which there were more upregulated genes than downregulated genes as compared to Mock untreated (NT). Heat map is shown from HOMER analysis with a cutoff of $\log _{2} \mathrm{FC} \geq 1$ representing genes with at least a twofold change in expression and an false discovery rate $(F D R) \leq 0.001$. Pathways are ordered by negative $\log 10 p$-values of enrichment. (B) Same as (A) except top 20 downregulated pathways are shown. (C) Representative scheme showing enrichment of the upregulated NF-KB survival signaling pathway after IRF5 knockdown. Boxes representing genes are color-coded based on the log2 fold-change values. Upregulated (red) or downregulated (blue) genes are shown as compared to Mock NT. Expression is shown by color code in four sections of each gene box. The gene box is divided into sections with one to one mapping comparisons of IRF5KD_NT, IRF5KD_anti-IgM+C CpG, Scr_anti-IgM+ $\mathrm{CpG}$, and Mock_anti-lgM+ $\mathrm{CpG}$ vs. reference Mock_NT, as shown in the Legend.

$(6,48)$. IRF5 is known to act downstream of TLR signaling in monocytes and dendritic cells, but characterization of IRF5 activation in human B cells has not been previously shown. Here we demonstrate that the transcription factor IRF5 acts downstream of TLR signaling to drive proliferation and differentiation to ASCs. However, unlike other transcription factors involved in ASC differentiation, such as IRF4, PRDM1, XBP1, and MYC that require early transcriptional upregulation (Figures 5E,F), IRF5 is uniquely poised for the rapid modulation of ASC-associated gene transcription in human $\mathrm{B}$ cells since its early regulation occurs through nuclear translocation (Figures 1A-C). Whether this effect is dependent on TLR9/BCR-induced B cell activation or would occur downstream of other B cell activating pathways is not currently known. However, since previous data in Irf5 $5^{-1-}$ mice showed a direct transcriptional role for IRF5 in the regulation of $P R D M 1$ and the $\gamma 2 a$ locus $(22,25)$, it is intriguing to consider IRF5 as a global mediator of antibody responses. Our finding of significantly reduced plasmablast differentiation and IgG antibody production following IRF5 knockdown highlights the role of IRF5 in mediating antibody responses. Contrary 


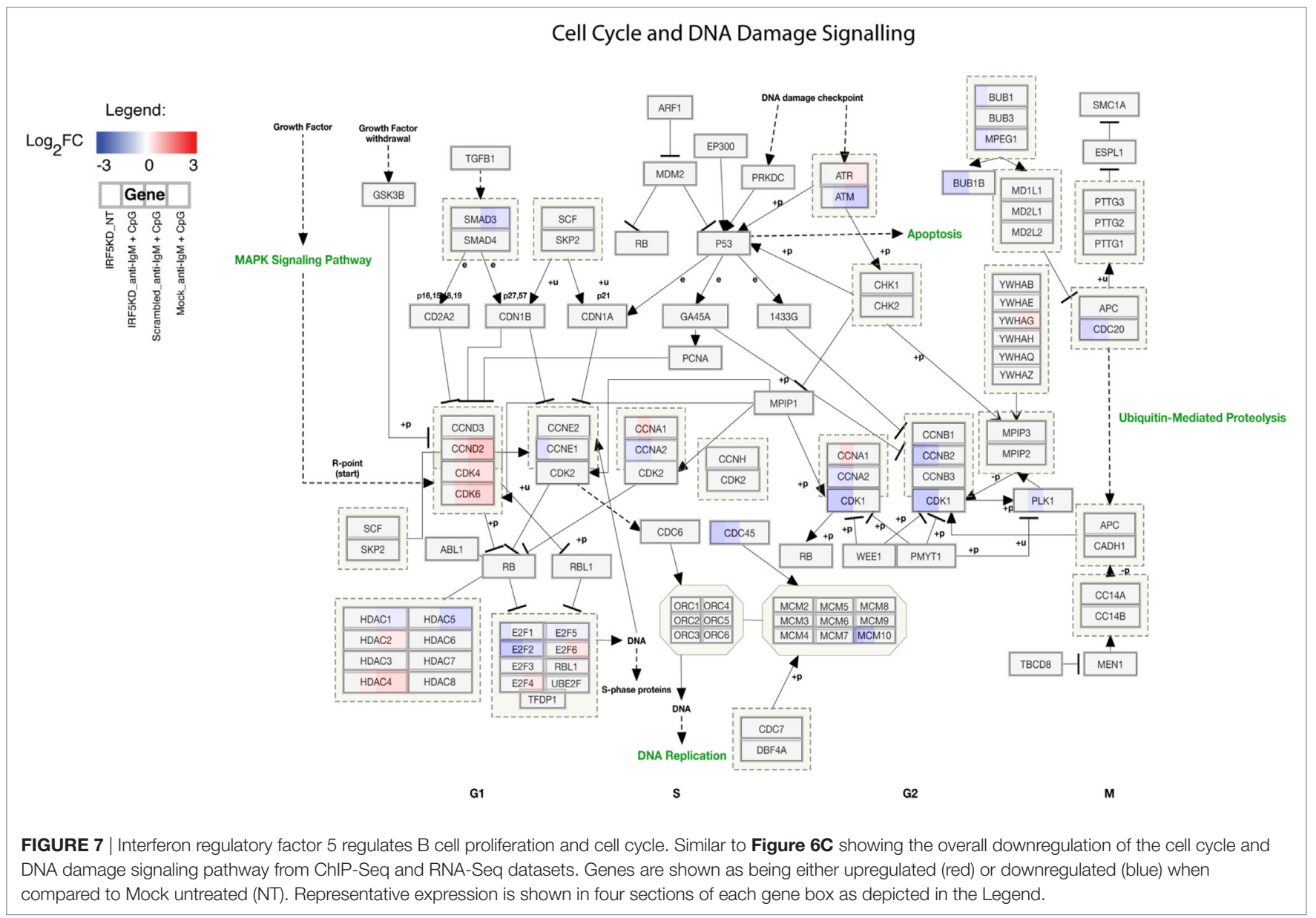

to murine data, however, we were unable to detect IRF5 peak enrichment at PRDM1 or any IgG locus by ChIP-Seq. This may be due to the early time point of analysis ( $4 \mathrm{~h}$ post-stimulation) or that these genes do not contain regulatory sequences recognized by IRF5. Similar to Irf5 $5^{-1-}$ mice, though, data presented in Figure 3G suggest a role for IRF5 in controlling IgG isotype secretion; however, IgG2 and 4 levels were secreted at significantly lower levels than IgG1 and 3 in response to stimulation. Additional work will be necessary to determine the role of IRF5 in human CSR.

The finding of reduced plasmablast differentiation in isolated naive B cells nucleofected with IRF5 siRNA highlights a new B cell-intrinsic role for IRF5. These findings overlap with recent work in a murine model of lupus showing reduced ASCs in $\operatorname{Irf5} 5^{-/-} \mathrm{MRL} / \mathrm{lpr}$ mice (20). However, it was not determined whether reduced ASCs was a result of IRF5 B cell-intrinsic or -extrinsic function. Data presented herein also point to IRF5 influences on late stage B cell effector functions. This is supported by the large difference in IgG isotype secretion as compared to intracellular IgG suggesting a potential role for IRF5 in secretion (Figures 3D-F).

Interferon regulatory factor 5 activity/function following nuclear translocation can occur through either transcriptional regulation or protein-protein interactions. We conclude from
ChIP-Seq analysis that IRF5 regulates the transcriptional control of ASC differentiation. Through ChIP-Seq analysis, we found that IRF5 bound a wide range of target genes involved in proliferation, ASC differentiation, and cell cycle control. Among the most prominent targets were ERK1, IRF4, and MYC. Previous characterization of IRF5 transcriptional targets was in murine macrophages, revealing that IRF5 and NFkB bound promoters of inflammatory cytokines (60). Earlier ChIP-Seq work in human PBMC found IRF5 to have overlapping transcriptional targets with STAT4 (70). Results presented herein represent the first characterization of IRF5 target genes in human primary B cells. In stark contrast to previous work, IRF5 regulated pathways in human B cells are focused on proliferation, survival, cell cycle, and ASC differentiation (Figures 6 and 7; Figure S5 in Supplementary Material).

Results from RNA-Seq further confirm an intrinsic role for IRF5 in ASC differentiation. Genes known to be critical in B cell proliferation and differentiation were drastically reduced following knockdown (Figure 5F). Our finding of decreased PRDM1 expression was similar to previous work in $\operatorname{Irf5} 5^{-/-}$mice carrying mutant DOCK2 $(25,26)$; however, we did not detect direct binding of IRF5 to the PRDM1 promoter even though ISRE and IRF-E sites were identified (Table 3). Rather, data indicate that decreased PRDM1 expression is a byproduct of other genes 
regulated by IRF5, such as $E R K 1$ and $M Y C(8,62)$. It is intriguing to propose from these data that IRF5 may be a gatekeeper for antigen-stimulated naive B cells to enter the proliferation cycle. If true, then alterations in IRF5 activation during early stages of $B$ cell activation may initiate the first break in self-tolerance. Current limitations in manipulating primary B cells, however, did not allow us to address whether the defect in B cell proliferation was due to reduced proliferation potential or reduced numbers of cells entering the cell cycle (Figures 3A,B). However, these data provide insight into other aspects of IRF5 function. For instance, the observed decrease in $\mathrm{B}$ cell proliferation suggests that the significant increase in $\operatorname{IgD}^{+} \mathrm{B}$ cells detected after IRF5 knockdown is due to retention of $\operatorname{IgD}^{+} \mathrm{B}$ cells rather than increased de novo synthesis since this would have been detected as an increase in proliferation rather than a decrease. Further, it implies that sufficient cell numbers were present for entering the cell cycle in response to BCR/TLR9 signaling. Instead, findings from pathway analysis of ChIP-Seq and RNA-Seq datasets support a key role for IRF5 in B cell proliferation via cell cycle regulation (Figure 7). Unfortunately, we were also unable to determine whether overexpression of MYC could restore ASC differentiation in IRF5 knockdown cells due to limitiations in transfection and/or viral infection efficiency $(54,55,71)$. Complementary experiments in mice or CRISPR/Cas9 in primary naive B cells (72) will be required to further elucidate the mechanisms by which IRF5 controls early stages of ASC differentiation.

Last, results presented herein have direct implications for IRF5 function in SLE B cells. SLE patients carrying IRF5 risk polymorphisms show elevated IRF5 expression in B lymphoblastoid cell lines $(15,16)$. SLE patients have elevated numbers of circulating ASCs that contribute to pathogenic autoantibody production and elevated circulating autoantibodies are present years before clinical symptoms $(2,4)$. It is intriguing to propose that polymorphisms in IRF5 contribute to early skewing of B cell subset distribution and to breaks in self-tolerance which may ultimately lead to the observed increase in circulating ASCs and autoantibodies found in SLE patients.

\section{REFERENCES}

1. Shapiro-Shelef M, Calame K. Regulation of plasma-cell development. Nat Rev Immunol (2005) 5:230-42. doi:10.1038/nri1572

2. Dorner T, Giesecke C, Lipsky PE. Mechanisms of B cell autoimmunity in SLE. Arthritis Res Ther (2011) 13:243. doi:10.1186/ar3433

3. Nutt SL, Hodgkin PD, Tarlinton DM, Corcoran LM. The generation of antibody-secreting plasma cells. Nat Rev Immunol (2015) 15:160-71. doi:10.1038/ nri3795

4. Dorner T, Jacobi AM, Lee J, Lipsky PE. Abnormalities of B cell subsets in patients with systemic lupus erythematosus. JImmunol Methods (2011) 363:187-97. doi:10.1016/j.jim.2010.06.009

5. Ruprecht CR, Lanzavecchia A. Toll-like receptor stimulation as a third signal required for activation of human naive B cells. Eur I Immunol (2006) 36:810-6. doi:10.1002/eji.200535744

6. Huggins J, Pellegrin T, Felgar RE, Wei C, Brown M, Zheng B, et al. CpG DNA activation and plasma-cell differentiation of CD27- naive human $B$ cells. Blood (2007) 109:1611-9. doi:10.1182/blood-2006-03-008441

7. Niiro H, Clark EA. Regulation of B-cell fate by antigen-receptor signals. Nat Rev Immunol (2002) 2:945. doi:10.1038/nri955

8. Yasuda T, Sanjo H, Pages G, Kawano Y, Karasuyama H, Pouyssegur J, et al. Erk kinases link pre-B cell receptor signaling to transcriptional events required

\section{ETHICS STATEMENT}

This study was carried out in accordance with the recommendations of the Rutgers Biomedical and Health Sciences IRB and the Feinstein Institute for Medical Research IRB with written informed consent from all subjects. All subjects gave written informed consent in accordance with the Declaration of Helsinki. The protocol was approved by the Rutgers Biomedical and Health Sciences IRB and the Feinstein Institute for Medical Research IRB.

\section{AUTHOR CONTRIBUTIONS}

SD and BB planned and designed the study. SD and TS performed the experiments. SD and $\mathrm{BZ}$ analyzed bioinformatics data. SD, SS, AW, BZ, RD, TS, and BB analyzed experimental data. $\mathrm{SD}$ and $\mathrm{BB}$ prepared the manuscript.

\section{ACKNOWLEDGMENTS}

We thank B. Diamond and members of the Barnes Lab for their insights and careful reading of the manuscript. We thank colleagues of the Rutgers flow cytometry and sequencing cores for technical assistance and the NYU Genome Center.

\section{FUNDING}

This work was supported by the National Institutes of Health grant AR065959-01 (BB), Alliance for Lupus Research (BB), and New Jersey Commission on Cancer Research (SD).

\section{SUPPLEMENTARY MATERIAL}

The Supplementary Material for this article can be found online at http://www.frontiersin.org/articles/10.3389/fimmu.2017.01938/ full\#supplementary-material.

for early B cell expansion. Immunity (2008) 28:499-508. doi:10.1016/j. immuni.2008.02.015

9. Hua Z, Hou B. TLR signaling in B-cell development and activation. Cell Mol Immunol (2013) 10:103-6. doi:10.1038/cmi.2012.61

10. Dominguez-Sola D, Victora GD, Ying CY, Phan RT, Saito M, Nussenzweig MC, et al. The proto-oncogene MYC is required for selection in the germinal center and cyclic reentry. Nat Immunol (2012) 13:1083-91. doi:10.1038/ ni. 2428

11. Calado DP, Sasaki Y, Godinho SA, Pellerin A, Kochert K, Sleckman BP, et al. The cell-cycle regulator $\mathrm{c}-\mathrm{Myc}$ is essential for the formation and maintenance of germinal centers. Nat Immunol (2012) 13:1092-100. doi:10.1038/ni.2418

12. Klein U, Casola S, Cattoretti G, Shen Q, Lia M, Mo T, et al. Transcription factor IRF4 controls plasma cell differentiation and class-switch recombination. Nat Immunol (2006) 7:773-82. doi:10.1038/ni1357

13. Shaffer AL, Lin KI, Kuo TC, Yu X, Hurt EM, Rosenwald A, et al. Blimp-1 orchestrates plasma cell differentiation by extinguishing the mature B cell gene expression program. Immunity (2002) 17:51-62. doi:10.1016/S10747613(02)00335-7

14. Shaffer AL, Shapiro-Shelef M, Iwakoshi NN, Lee AH, Qian SB, Zhao H, et al. XBP1, downstream of Blimp-1, expands the secretory apparatus and other organelles, and increases protein synthesis in plasma cell differentiation. Immunity (2004) 21:81-93. doi:10.1016/j.immuni.2004.06.010 
15. Graham RR, Kozyrev SV, Baechler EC, Reddy MV, Plenge RM, Bauer JW, et al. A common haplotype of interferon regulatory factor 5 (IRF5) regulates splicing and expression and is associated with increased risk of systemic lupus erythematosus. Nat Genet (2006) 38:550-5. doi:10.1038/ ng1782

16. Graham RR, Kyogoku C, Sigurdsson S, Vlasova IA, Davies LR, Baechler EC, et al. Three functional variants of IFN regulatory factor 5 (IRF5) define risk and protective haplotypes for human lupus. Proc Natl Acad Sci U S A (2007) 104:6758-63. doi:10.1073/pnas.0701266104

17. Sigurdsson S, Goring HH, Kristjansdottir G, Milani L, Nordmark G, Sandling JK, et al. Comprehensive evaluation of the genetic variants of interferon regulatory factor 5 (IRF5) reveals a novel 5 bp length polymorphism as strong risk factor for systemic lupus erythematosus. Hum Mol Genet (2008) 17:872-81. doi:10.1093/hmg/ddm359

18. Feng D, Stone RC, Eloranta ML, Sangster-Guity N, Nordmark G, Sigurdsson S, et al. Genetic variants and disease-associated factors contribute to enhanced interferon regulatory factor 5 expression in blood of patients with systemic lupus erythematosus. Arthritis Rheum (2010) 62:562-73. doi:10.1002/ art. 27223

19. Stone RC, Feng D, Deng J, Singh S, Yang L, Fitzgerald-Bocarsly P, et al. Interferon regulatory factor 5 activation in monocytes of systemic lupus erythematosus patients is triggered by circulating autoantigens independent of type I interferons. Arthritis Rheum (2012) 64:788-98. doi:10.1002/ art.33395

20. Yasuda K, Watkins AA, Kochar GS, Wilson GE, Laskow B, Richez C, et al. Interferon regulatory factor-5 deficiency ameliorates disease severity in the MRL/lpr mouse model of lupus in the absence of a mutation in DOCK2. PLoS One (2014) 9:e103478. doi:10.1371/journal.pone.0103478

21. Feng D, Yang L, Bi X, Stone RC, Patel P, Barnes BJ. Irf5-deficient mice are protected from pristane-induced lupus via increased Th2 cytokines and altered IgG class switching. Eur J Immunol (2012) 42:1477-87. doi:10.1002/ eji.201141642

22. Savitsky DA, Yanai H, Tamura T, Taniguchi T, Honda K. Contribution of IRF5 in B cells to the development of murine SLE-like disease through its transcriptional control of the IgG2a locus. Proc Natl Acad Sci U S A (2010) 107:10154-9. doi:10.1073/pnas.1005599107

23. Xu WD, Pan HF, Xu Y, Ye DQ. Interferon regulatory factor 5 and autoimmune lupus. Expert Rev Mol Med (2013) 15:e6. doi:10.1017/erm.2013.7

24. Takaoka A, Yanai H, Kondo S, Duncan G, Negishi H, Mizutani T, et al. Integral role of IRF- 5 in the gene induction programme activated by toll-like receptors. Nature (2005) 434:243-9. doi:10.1038/nature03308

25. Lien C, Fang CM, Huso D, Livak F, Lu R, Pitha PM. Critical role of IRF-5 in regulation of B-cell differentiation. Proc Natl Acad Sci US A (2010) 107:4664-8. doi:10.1073/pnas.0911193107

26. Purtha WE, Swiecki M, Colonna M, Diamond MS, Bhattacharya D. Spontaneous mutation of the Dock2 gene in Irf5-/- mice complicates interpretation of type I interferon production and antibody responses. Proc Natl Acad Sci U S A (2012) 109:E898-904. doi:10.1073/pnas.1118155109

27. Chan VS, Tsang HH, Tam RC, Lu L, Lau CS. B-cell-targeted therapies in systemic lupus erythematosus. Cell Mol Immunol (2013) 10:133-42. doi:10.1038/ cmi.2012.64

28. Bergstrom B, Aune MH, Awuh JA, Kojen JF, Blix KJ, Ryan L, et al. TLR8 senses Staphylococcus aureus RNA in human primary monocytes and macrophages and induces IFN-beta production via a TAK1-IKKbeta-IRF5 signaling pathway. J Immunol (2015) 195:1100-11. doi:10.4049/jimmunol. 1403176

29. del Fresno C, Soulat D, Roth S, Blazek K, Udalova I, Sancho D, et al. Interferonbeta production via Dectin-1-Syk-IRF5 signaling in dendritic cells is crucial for immunity to C. albicans. Immunity (2013) 38:1176-86. doi:10.1016/j. immuni.2013.05.010

30. Griesbeck M, Ziegler S, Laffont S, Smith N, Chauveau L, Tomezsko P, et al. Sex differences in plasmacytoid dendritic cell levels of IRF5 drive higher IFNalpha production in women. J Immunol (2015) 195:5327-36. doi:10.4049/ jimmunol.1501684

31. Hedl M, Abraham C. IRF5 risk polymorphisms contribute to interindividual variance in pattern recognition receptor-mediated cytokine secretion in human monocyte-derived cells. J Immunol (2012) 188:5348-56. doi:10.4049/ jimmunol.1103319
32. Hedl M, Yan J, Abraham C. IRF5 and IRF5 disease-risk variants increase glycolysis and human M1 macrophage polarization by regulating proximal signaling and Akt2 activation. Cell Rep (2016) 16:2442-55. doi:10.1016/j. celrep.2016.07.060

33. Krausgruber T, Blazek K, Smallie T, Alzabin S, Lockstone H, Sahgal N, et al. IRF5 promotes inflammatory macrophage polarization and TH1-TH17 responses. Nat Immunol (2011) 12:231-8. doi:10.1038/ni.1990

34. Steinhagen F, Rodriguez LG, Tross D, Tewary P, Bode C, Klinman DM. IRF5 and IRF8 modulate the CAL-1 human plasmacytoid dendritic cell line response following TLR9 ligation. Eur J Immunol (2016) 46:647-55. doi:10.1002/eji.201545911

35. Pelka K, Latz E. IRF5, IRF8, and IRF7 in human pDCs - the good, the bad, and the insignificant? Eur J Immunol (2013) 43:1693-7. doi:10.1002/eji. 201343739

36. Li D, De S, Li D, Song S, Matta B, Barnes BJ. Specific detection of interferon regulatory factor 5 (IRF5): a case of antibody inequality. Sci Rep (2016) 6:31002. doi:10.1038/srep31002

37. Yan H, Evans J, Kalmbach M, Moore R, Middha S, Luban S, et al. HiChIP: a high-throughput pipeline for integrative analysis of ChIP-Seq data. BMC Bioinformatics (2014) 15:280. doi:10.1186/1471-2105-15-280

38. Li H, Durbin R. Fast and accurate short read alignment with Burrows-Wheeler transform. Bioinformatics (2009) 25:1754-60. doi:10.1093/bioinformatics/ btp324

39. Zhang Y, Liu T, Meyer CA, Eeckhoute J, Johnson DS, Bernstein BE, et al. Model-based analysis of ChIP-Seq (MACS). Genome Biol (2008) 9:R137. doi:10.1186/gb-2008-9-9-r137

40. Thorvaldsdottir H, Robinson JT, Mesirov JP. Integrative genomics viewer (IGV): high-performance genomics data visualization and exploration. Brief Bioinform (2013) 14:178-92. doi:10.1093/bib/bbs017

41. Dobin A, Davis CA, Schlesinger F, Drenkow J, Zaleski C, Jha S, et al. STAR: ultrafast universal RNA-seq aligner. Bioinformatics (2013) 29:15-21. doi:10.1093/bioinformatics/bts635

42. Liao Y, Smyth GK, Shi W. featureCounts: an efficient general purpose program for assigning sequence reads to genomic features. Bioinformatics (2014) 30:923-30. doi:10.1093/bioinformatics/btt656

43. Zhao S, Xi L, Quan J, Xi H, Zhang Y, von Schack D, et al. QuickRNASeq lifts large-scale RNA-seq data analyses to the next level of automation and interactive visualization. BMC Genomics (2016) 17:3. doi:10.1186/s12864015-2356-9

44. Robinson MD, McCarthy DJ, Smyth GK. edgeR: a Bioconductor package for differential expression analysis of digital gene expression data. Bioinformatics (2010) 26:139-40. doi:10.1093/bioinformatics/btp616

45. Conesa A, Madrigal P, Tarazona S, Gomez-Cabrero D, Cervera A, McPherson A, et al. A survey of best practices for RNA-seq data analysis. Genome Biol (2016) 17:13. doi:10.1186/s13059-016-0881-8

46. Heinz S, Benner C, Spann N, Bertolino E, Lin YC, Laslo P, et al. Simple combinations of lineage-determining transcription factors prime cis-regulatory elements required for macrophage and B cell identities. Mol Cell (2010) 38:576-89. doi:10.1016/j.molcel.2010.05.004

47. Kutmon M, Riutta A, Nunes N, Hanspers K, Willighagen EL, Bohler A, et al. WikiPathways: capturing the full diversity of pathway knowledge. Nucleic Acids Res (2016) 44:D488-94. doi:10.1093/nar/gkv1024

48. Genestier L, Taillardet M, Mondiere P, Gheit H, Bella C, Defrance T. TLR agonists selectively promote terminal plasma cell differentiation of B cell subsets specialized in thymus-independent responses. JImmunol (2007) 178:7779-86. doi:10.4049/jimmunol.178.12.7779

49. Barnes BJ, Moore PA, Pitha PM. Virus-specific activation of a novel interferon regulatory factor, IRF-5, results in the induction of distinct interferon alpha genes. J Biol Chem (2001) 276:23382-90. doi:10.1074/jbc. M101216200

50. Barnes BJ, Kellum MJ, Field AE, Pitha PM. Multiple regulatory domains of IRF-5 control activation, cellular localization, and induction of chemokines that mediate recruitment of T lymphocytes. Mol Cell Biol (2002) 22:5721-40. doi:10.1128/MCB.22.16.5721-5740.2002

51. Schoenemeyer A, Barnes BJ, Mancl ME, Latz E, Goutagny N, Pitha PM, et al. The interferon regulatory factor, IRF5, is a central mediator of toll-like receptor 7 signaling. J Biol Chem (2005) 280:17005-12. doi:10.1074/jbc. M412584200 
52. Bernasconi NL, Onai N, Lanzavecchia A. A role for toll-like receptors in acquired immunity: up-regulation of TLR9 by BCR triggering in naïve B cells and constitutive expression in memory B cells. Blood (2003) 101:4500-4. doi:10.1182/blood-2002-11-3569

53. Blanchard-Rohner G, Pulickal AS, Jol-van der Zijde CM, Snape MD, Pollard AJ. Appearance of peripheral blood plasma cells and memory B cells in a primary and secondary immune response in humans. Blood (2009) 114:4998-5002. doi:10.1182/blood-2009-03-211052

54. Kardava L, Moir S, Wang W, Ho J, Buckner CM, Posada JG, et al. Attenuation of HIV-associated human B cell exhaustion by siRNA downregulation of inhibitory receptors. J Clin Invest (2011) 121:2614-24. doi:10.1172/JCI45685

55. Seiffert M, Stilgenbauer S, Dohner H, Lichter P. Efficient nucleofection of primary human B cells and B-CLL cells induces apoptosis, which depends on the microenvironment and on the structure of transfected nucleic acids. Leukemia (2007) 21:1977-83. doi:10.1038/sj.leu.2404863

56. Hu G, Mancl ME, Barnes BJ. Signaling through IFN regulatory factor-5 sensitizes p53-deficient tumors to DNA damage-induced apoptosis and cell death. Cancer Res (2005) 65:7403-12. doi:10.1158/0008-5472.CAN-05-0583

57. Hu G, Barnes BJ. IRF-5 is a mediator of the death receptor-induced apoptotic signaling pathway. J Biol Chem (2009) 284:2767-77. doi:10.1074/jbc. M804744200

58. Agrawal S, Gupta S. TLR1/2, TLR7, and TLR9 signals directly activate human peripheral blood naive and memory B cell subsets to produce cytokines, chemokines, and hematopoietic growth factors. JClin Immunol (2011) 31:89-98. doi:10.1007/s10875-010-9471-9

59. Liu BS, Stoop JN, Huizinga TW, Toes RE. IL-21 enhances the activity of the TLR-MyD88-STAT3 pathway but not the classical TLR-MyD88-NF-kappaB pathway in human B cells to boost antibody production. J Immunol (2013) 191:4086-94. doi:10.4049/jimmunol.1300765

60. Saliba DG, Heger A, Eames HL, Oikonomopoulos S, Teixeira A, Blazek K, et al. IRF5:RelA interaction targets inflammatory genes in macrophages. Cell Rep (2014) 8:1308-17. doi:10.1016/j.celrep.2014.07.034

61. Leifer CA, Kennedy MN, Mazzoni A, Lee C, Kruhlak MJ, Segal DM. TLR9 is localized in the endoplasmic reticulum prior to stimulation. J Immunol (2004) 173:1179-83. doi:10.4049/jimmunol.173.2.1179

62. Gold MR. B cell development: important work for ERK. Immunity (2008) 28:488-90. doi:10.1016/j.immuni.2008.03.008

63. Yeo L, Lom H, Juarez M, Sonw M, Buckley CD, Filer A, et al. Expression of FcRL4 defines a pro-inflammatory, RANKL-producing B cell subset in rheumatoid arthritis. Ann Rheum Dis (2015) 74:928-35. doi:10.1136/ annrheumdis-2013-204116
64. Cantor J, Browne CD, Ruppert R, Feral CC, Fassler R, Rickert RC, et al. CD98hc facilitates B cell proliferation and adaptive humoral immunity. Nat Immunol (2009) 10:412-9. doi:10.1038/ni.1712

65. Cuylen S, Blaukopf C, Politi AZ, Muller-Reichert T, Neumann B, Poser I, et al. Ki-67 acts as a biological surfactant to disperse mitotic chromosomes. Nature (2016) 535:308-12. doi:10.1038/nature18610

66. Chen S, Miyazaki M, Chandra V, Fisch KM, Chang AN, Murre C. Id3 orchestrates germinal center B cell development. Mol Cell Biol (2016) 36:2543-52. doi:10.1128/MCB.00150-16

67. Gloury R, Zotos D, Zuidscherwoude M, Masson F, Liao Y, Hasbold J, et al. Dynamic changes in Id3 and E-protein activity orchestrate germinal center and plasma cell development. J Exp Med (2016) 213:1095-111. doi:10.1084/ jem.20152003

68. Park K, Nowyhed H, Hedrick C. Nr4al (Nur77) regulates B cell survival and activation. (P1453). J Immunol (2013) 190:174-9.

69. Heise N, De Silva NS, Silva K, Carette A, Simonetti G, Pasparakis M, et al. Germinal center B cell maintenance and differentiation are controlled by distinct NF-kB transcription factor subunits. J Exp Med (2014) 211:2103-18. doi:10.1084/jem.20132613

70. Wang C, Sandling JK, Hagberg N, Berggren O, Sigurdsson S, Karlberg O, et al. Genome-wide profiling of target genes for the systemic lupus erythematosus-associated transcription factors IRF5 and STAT4. Ann Rheum Dis (2013) 72:96-103. doi:10.1136/annrheumdis-2012-201364

71. Amirache F, Levy C, Costa C, Mangeot P-E, Torbett BE, Wang CX, et al. Mystery solved: VSV-G-LVs do not allow efficient gene transfer into unstimulated T cells, B cells, and HSCs because they lack the LDL receptor. Blood (2014) 123:1422-4. doi:10.1182/blood-2013-11-540641

72. Chu VT, Graf R, Rajewsky K. CRISPR/Cas9-mediated in vitro mutagenesis in GC-like B cells. Methods Mol Biol (2017) 1623:135-45. doi:10.1007/978-1-4939-7095-7_12

Conflict of Interest Statement: BZ and AW were employed by Pfizer Inc. All other authors declare not competing interests.

Copyright (C) 2018 De, Zhang, Shih, Singh, Winkler, Donnelly and Barnes. This is an open-access article distributed under the terms of the Creative Commons Attribution License (CC BY). The use, distribution or reproduction in other forums is permitted, provided the original author(s) or licensor are credited and that the original publication in this journal is cited, in accordance with accepted academic practice. No use, distribution or reproduction is permitted which does not comply with these terms. 